\title{
Topological Multiband s-wave Superconductivity in Coupled Multifold Fermions
}

\author{
Changhee Lee, ${ }^{1}$ Chiho Yoon, ${ }^{1}$ Taehyeok Kim, ${ }^{1}$ Suk Bum Chung, $, 2,3,4, *$ and Hongki Min ${ }^{1, \dagger}$ \\ ${ }^{1}$ Department of Physics and Astronomy, Seoul National University, Seoul 08826, Korea \\ ${ }^{2}$ Department of Physics, University of Seoul, Seoul 02504, Korea \\ ${ }^{3}$ Natural Science Research Institute, University of Seoul, Seoul 02504, Korea \\ ${ }^{4}$ School of Physics, Korea Institute for Advanced Study, Seoul 02455, Korea
}

(Dated: January 11, 2022)

\begin{abstract}
We study three-dimensional time-reversal-invariant topological superconductivity in noncentrosymmetric materials such as $\mathrm{RhSi}$, CoSi, and AlPt which host coupled multifold nodes energetically split by the spin-orbit coupling at the same time-reversal-invariant momentum (TRIM). The topological superconductivity arises from the $s_{+} \oplus s_{-}$gap function, which is $\boldsymbol{k}$ independent, but with opposite signs for the two nodes split at the same TRIM. We consider various electron-electron interactions in the tight-binding model for $\mathrm{RhSi}$ and find that the topological superconducting phase supporting a surface Majorana cone and topological nodal rings is favored in a wide range of interaction parameters.
\end{abstract}

Introduction.- Majorana particles on superconductors have drawn widespread attention as an avenue to topological quantum computation [1-5], and this can be attributed to their characteristic non-abelian vortex exchange statistics [6-8]. The majority of experimental search for the Majorana zero modes (MZMs) in condensed matter physics has been based on proposals to realize them in a heterostructure. Examples include the semiconductor nanowire [9-13], the ferromagnetic atomic chain [14], and the topological insulator $[15,16]$ in proximity to a conventional superconductor.

Besides these heterostructures, the development of topological band theory [17-19] has demonstrated that topological materials could exhibit topological superconductivity (TSC) with Majorana boundary modes due to the novel interplay between the unique electronic structures and interactions [20-25]. Recent research has been devoted to understanding the interplay and the resulting TSC in Weyl/Dirac semimetals [26-29] and Luttinger semimetals [30, 31].

The recently discovered topological semimetals with unconventional multifold fermions [32] also provide an ideal platform for studying the effects of interactions in the unique electronic structures, especially TSC. In a family of candidate materials with the B20 crystal structure [33, 34] such as RhSi, CoSi and AlPt, the topological features of the electronic structure such as the long surface Fermi arcs have been observed [35-41]. However, some qualitative band structure features of this class of candidate materials have not been included in the existing studies on the interacting multifold fermions [4246]. Many of them studied isolated multifold fermions at a single time-reversal invariant momentum (TRIM) and it has not been considered that two types of multifold fermions lie at slightly different energy levels on the same TRIM. Thus, the possibility of the multi-band characteristics to manifest in the superconductivity [4750] has been overlooked.

In this Letter, we study a possible time-reversal in- variant (TRI) TSC arising from the coupled structure of multifold fermions at TRIMs in a representative multifold fermion system, RhSi. We find that the electronic structure of coupled multifold fermions can give rise to a robust combination of the fully gapped TRI TSC around the coupled nodes at $\Gamma$ and the nodal TSC around another TRIM, which arises from the $\boldsymbol{k}$-independent intranodal pairing gap at each TRIM with opposite signs between the two nodes at the same TRIM; we shall call this the " $s_{+} \oplus s_{-}$gap function".

Low energy effective model. - To study the electronic structure of RhSi, we adopt the tight-binding (TB) model proposed in Ref. [35] (see Sec. I of Supplemental Material (SM) [51] for the details). Figure 1(a) shows the $\mathrm{TB}$ band structure of RhSi and the first Brillouin zone (BZ) along with the surface BZ (SBZ) on the (001) plane. At $\Gamma$, four-fold and two-fold nodal points are found around the zero energy corresponding to the (pseudo)spin- $\frac{3}{2}$ Rarita-Schwinger-Weyl (RSW) fermion and spin- $\frac{1}{2}$ Kramer-Weyl (KW) fermion, respectively. Additionally, there are six-fold and two-fold nodal points at $\mathrm{R}$ which are equivalent to doubly degenerate spin-1 $[39,53-55]$ and spin-0 systems, respectively. As shown in Fig. 1(a), the Fermi level of RhSi lies around the fourfold nodal point at $\Gamma$ and the energy bands crossing the Fermi level emanate from the multifold nodal points at $\Gamma$ and $\mathrm{R}$.

We first investigate a low-energy effective model around $\Gamma$. Up to first order in $\boldsymbol{k}$, the electronic band structure near the two nodal points at $\Gamma$ is described by the following Hamiltonian:

$$
H_{\boldsymbol{k}}^{\frac{3}{2} \oplus \frac{1}{2}}=\left(\begin{array}{cc}
H_{\boldsymbol{k}}^{\left(\frac{3}{2}\right)} & T_{\Gamma, \boldsymbol{k}} \\
T_{\Gamma, \boldsymbol{k}}^{\dagger} & H_{\boldsymbol{k}}^{\left(\frac{1}{2}\right)}-m_{\Gamma} I_{2}
\end{array}\right),
$$

where $H_{\boldsymbol{k}}^{\left(\frac{3}{2}\right)}=v_{\Gamma} \boldsymbol{k} \cdot \boldsymbol{S}^{\left(\frac{3}{2}\right)}$ and $H_{\boldsymbol{k}}^{\left(\frac{1}{2}\right)}=2 v_{\Gamma} \boldsymbol{k} \cdot \boldsymbol{S}^{\left(\frac{1}{2}\right)}$ describe the RSW and KW fermions, respectively, with the spin- $s$ matrices $S_{i}^{(s)}$ satisfying $\boldsymbol{S}^{(s) 2}=s(s+1)$. Here, $v_{\Gamma}$ and $2 v_{\Gamma}$ are the Fermi velocities of RSW and KW fermions, 




(b)


Figure 1. (a) Band structure of RhSi along the high-symmetry lines in the BZ. The BZ and the (001) SBZ are shown in the inset. (b),(c) The band structure of the continuum model near the nodal points at $\Gamma(\mathrm{R})$. The ratio of the wave function magnitude between $\left|\frac{3}{2}, \frac{1}{2}, \hat{\boldsymbol{k}}\right\rangle$ and $\left|\frac{1}{2}, \frac{1}{2}, \hat{\boldsymbol{k}}\right\rangle(|1,0, \hat{\boldsymbol{k}}\rangle$ and $|0,0, \hat{\boldsymbol{k}}\rangle)$ is illustrated by different colors. For the bands with $E>0, \mathrm{Ch}_{\text {in }}$ and $\mathrm{Ch}_{\text {out }}$ are the Chern numbers carried by the inner and outer FSSs, respectively, and $\times 2$ in the subscript indicates double degeneracy.

respectively. In RhSi family, they originate from spinindependent inversion-breaking hoppings. We denote the eigenstates of $H_{k}^{(s)}$ by $\left|s, h_{z}, \hat{\boldsymbol{k}}\right\rangle$, where $s$ and $h_{z}$ are the total angular momentum and the helicity eigenvalue, respectively. We assume $v_{\Gamma}>0$ without loss of generality. The two nodal points are energetically separated by $m_{\Gamma}$ and coupled by $T_{\Gamma, \boldsymbol{k}}=\frac{v_{\Gamma} k}{\sqrt{2}}\left(\left|\frac{3}{2}, \frac{1}{2}, \hat{\boldsymbol{k}}\right\rangle\left\langle\frac{1}{2}, \frac{1}{2}, \hat{\boldsymbol{k}}\right|+\right.$ $\left.\left|\frac{3}{2},-\frac{1}{2}, \hat{\boldsymbol{k}}\right\rangle\left\langle\frac{1}{2},-\frac{1}{2}, \hat{\boldsymbol{k}}\right|\right)$.

When the Fermi level lies above the four-fold nodal point, two bands of $H_{\boldsymbol{k}}^{\frac{3}{2} \oplus \frac{1}{2}}$ cross the Fermi level as shown in Fig. 1(b). Their eigenstates are given by

$$
\begin{aligned}
\mid \Gamma, \text { in }, \boldsymbol{k}\rangle & =\left|\frac{3}{2}, \frac{3}{2}, \hat{\boldsymbol{k}}\right\rangle, \\
\mid \Gamma, \text { out }, \boldsymbol{k}\rangle & =\cos \theta_{\Gamma, k}\left|\frac{3}{2}, \frac{1}{2}, \hat{\boldsymbol{k}}\right\rangle+\sin \theta_{\Gamma, k}\left|\frac{1}{2}, \frac{1}{2}, \hat{\boldsymbol{k}}\right\rangle,
\end{aligned}
$$

where $\theta_{\Gamma, k}=\tan ^{-1} \sqrt{\frac{f_{\Gamma, k}-e_{\Gamma, k}}{f_{\Gamma, k}+e_{\Gamma, k}}}$ with $e_{\Gamma, k}=m_{\Gamma}-v_{\Gamma} k / 2$ and $f_{\Gamma, k}=\sqrt{e_{\Gamma, k}^{2}+2 v_{\Gamma}^{2} k^{2}}$. Here, "in" and "out" represent the inner and outer Fermi surface sheets (FSSs), respectively. Note that the outer FSS mainly consists of the $h_{z}=\frac{1}{2}$ branch of the RSW fermion in the limit
$v_{\Gamma} k \ll m_{\Gamma}$, while the portion of the KW fermion $\left|\frac{1}{2}, \frac{1}{2}, \hat{\boldsymbol{k}}\right\rangle$ increases to $\frac{2}{3}$ in the opposite limit. In Fig. 1(b), the bands composed of the $h_{z}=\frac{1}{2}$ branches of the RSW and KW fermions are colored according to their ratio. A FSS surrounding a multifold fermion carries a quantized Chern number $2 h_{z}$ when each eigenstate on the FSS is a superposition of $\left|s, h_{z}, \hat{\boldsymbol{k}}\right\rangle$ 's with the same $h_{z}$. Thus, the inner and outer FSSs carry the Chern numbers 3 and 1, respectively.

The low-energy effective model near $\mathrm{R}$ is analogous to that near $\Gamma$. Up to first order in $\boldsymbol{k}$, the electronic band structure around six-fold and two-fold nodal points at $\mathrm{R}$ is described by two copies of coupled spin- 1 and spin- 0 fermions $H_{\boldsymbol{k}}^{1 \oplus 0} \oplus H_{\boldsymbol{k}}^{1 \oplus 0}$, where $H_{\boldsymbol{k}}^{1 \oplus 0}$ is given by

$$
H_{\boldsymbol{k}}^{1 \oplus 0}=\left(\begin{array}{ll}
H_{\boldsymbol{k}}^{(1)} & T_{\mathrm{R}, \boldsymbol{k}} \\
T_{\mathrm{R}, \boldsymbol{k}}^{\dagger} & H_{\boldsymbol{k}}^{(0)}
\end{array}\right)=\left(\begin{array}{cc}
-v_{\mathrm{R}} \boldsymbol{k} \cdot \boldsymbol{S}^{(1)} & T_{\mathrm{R}, \boldsymbol{k}} \\
T_{\mathrm{R}, \boldsymbol{k}}^{\dagger} & -m_{\mathrm{R}}
\end{array}\right),
$$

where the two nodal points are coupled by $T_{\mathrm{R}, \boldsymbol{k}}=$ $v_{\mathrm{R}} k|1,0, \hat{\boldsymbol{k}}\rangle\langle 0,0, \hat{\boldsymbol{k}}|$. Here, $v_{\mathrm{R}}>0$ since the NielsenNinomiya theorem [56] requires $v_{\Gamma} v_{\mathrm{R}}>0$.

As shown in Fig. 1(c), two bands of $H_{k}^{1 \oplus 0}$ cross the Fermi level when it lies above the six-fold nodal point, whose eigenstates are given by

$$
\begin{aligned}
\mid \mathrm{R}, \text { in, } \boldsymbol{k}\rangle & =|1,-1, \hat{\boldsymbol{k}}\rangle, \\
\mid \mathrm{R}, \text { out }, \boldsymbol{k}\rangle & =\cos \theta_{\mathrm{R}, k}|1,0, \hat{\boldsymbol{k}}\rangle+\sin \theta_{\mathrm{R}, k}|0,0, \hat{\boldsymbol{k}}\rangle,
\end{aligned}
$$

where $\theta_{\mathrm{R}, k}=\tan ^{-1}\left(\sqrt{\frac{f_{\mathrm{R}, k}-m_{\mathrm{R}}}{f_{\mathrm{R}, k}+m_{\mathrm{R}}}}\right)$ with $f_{\mathrm{R}, k}=$ $\sqrt{m_{\mathrm{R}}^{2}+4 v_{\mathrm{R}}^{2} k^{2}}$. Eq. (4) is very similar to Eq. (2). The Chern numbers carried by the inner and outer FSSs are -2 and 0 , respectively, for each copy. Then the total Chern number of the Fermi surface around $\mathrm{R}$ is -4 compensating the Chern numbers from the FSSs around the RSW and KW nodes at $\Gamma$.

TSC from $s_{+} \oplus s_{-}$pairings. - Considering the two coupled nodal points at $\Gamma$ or R, TRI TSC can arise even from the simplest $\boldsymbol{k}$-independent gap functions represented by a direct sum of two trivial matrices respecting all the spatial symmetries of the system. Besides the trivial gap function, the multinodal nature of each TRIM in the system allows the $s_{+} \oplus s_{-}$gap functions:

$$
\Delta_{ \pm}^{\Gamma}=\left(\begin{array}{ll}
I_{4} & \\
& -2 I_{2}
\end{array}\right), \quad \Delta_{ \pm}^{\mathrm{R}}=\left(\begin{array}{ll}
I_{3} & \\
& -3 I_{1}
\end{array}\right),
$$

where $I_{2 s+1}$ acts on spin- $s$ fermions. $\Delta_{ \pm}^{\Gamma(\mathrm{R})}$ looks like the trivial gap function if we just focus on one of the two nodal points at $\Gamma(\mathrm{R})$, but the sign of the gap function on each nodal point is opposite. Note that the $s_{+} \oplus s_{-}$ gap functions indicate spin-triplet pairing (see Sec. II of SM [51]). We shall show later that, for generic electronelectron interactions, the TRI solution of the linearized gap equation can be written as

$$
\begin{aligned}
\Delta_{\Gamma}\left(\beta_{\Gamma}\right) & =\alpha_{\Gamma}\left(\Delta_{ \pm}^{\Gamma} \cos \beta_{\Gamma}+I_{6} \sin \beta_{\Gamma}\right), \\
\Delta_{\mathrm{R}}\left(\beta_{\mathrm{R}}\right) & =\alpha_{\mathrm{R}}\left(\Delta_{ \pm}^{\mathrm{R}} \cos \beta_{\mathrm{R}}+I_{4} \sin \beta_{\mathrm{R}}\right),
\end{aligned}
$$


(a)

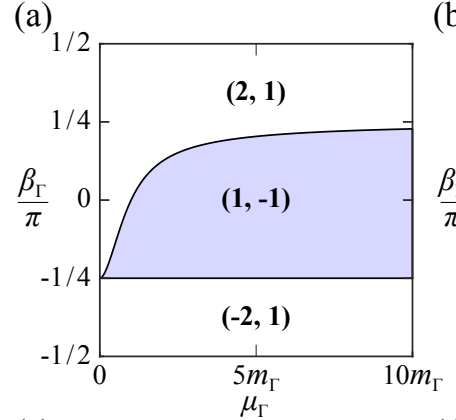

(b)


(d)

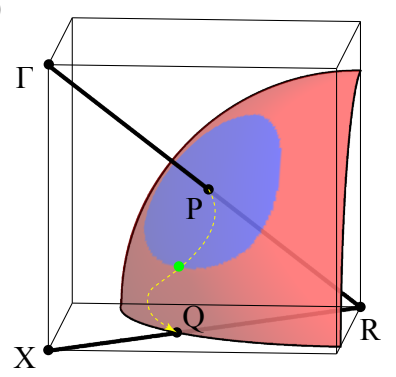

Figure 2. (a),(b) Phase diagram of the TSC in RhSi with respect to $\beta_{\Gamma}$ and $\mu_{\Gamma}$ with $\alpha_{\Gamma}>0\left(\beta_{\mathrm{R}}\right.$ and $\mu_{\mathrm{R}}$ with $\left.\alpha_{\mathrm{R}}>0\right)$. A pair of winding numbers $\left(w_{3 \mathrm{D}, \Gamma(\mathrm{R})}, w_{1 \mathrm{D}, \Gamma(\mathrm{R})}\right)$ on each region characterizes the topology of the superconducting phase. $w_{3 \mathrm{D}, \mathrm{R}}=\varnothing$ indicates the TSC with NRs in the bulk BdG spectrum. The dashed line represents $\mu_{\mathrm{R}}=E_{c 1}\left(\beta_{\mathrm{R}}\right)$ on which the superconducting gap closes/reopens without changing $w_{3 \mathrm{D}}$ and $w_{1 \mathrm{D}}$. (c) The band structure around $\mathrm{R}$ above the sixfold nodal point colored in red (blue) for positive (negative) $\delta_{\mathrm{R}, n}$. The four bands are labeled as $n=1,2,3,4$ in the order of closeness to R. (d) The FSS formed by band 2 at $\mu=\mu_{2}$, colored in the same manner as (c).

near $\Gamma$ and $\mathrm{R}$, respectively. Here, $\alpha_{\Gamma(\mathrm{R})}$ corresponds to the overall magnitude of the gap function and $\beta_{\Gamma(\mathrm{R})} \in$ $\left[-\frac{\pi}{2}, \frac{\pi}{2}\right]$ parameterizes the ratio between the trivial gap function and the $s_{+} \oplus s_{-}$gap function. We assume $\alpha_{\Gamma}, \alpha_{\mathrm{R}}>0$ here (see Sec. III of SM [51] for more general cases).

To understand the topological nature of the superconducting phase with the gap function $\Delta_{\Gamma}\left(\beta_{\Gamma}\right)$, we first focus on the multifold fermions at $\Gamma$ and study the Bogliubov-de Gennes (BdG) Hamiltonian $\hat{H}_{\mathrm{BdG}}=$ $\frac{1}{2} \sum_{\boldsymbol{k}} \hat{\Psi}_{\boldsymbol{k}}^{\dagger} H_{\mathrm{BdG}}(\boldsymbol{k}) \hat{\Psi}_{\boldsymbol{k}}$ with

$$
H_{\mathrm{BdG}, \Gamma}(\boldsymbol{k})=\left(\begin{array}{cc}
H_{\boldsymbol{k}}^{\frac{3}{2} \oplus \frac{1}{2}}-\mu_{\Gamma} & \Delta_{\Gamma}\left(\beta_{\Gamma}\right) \\
\Delta_{\Gamma}\left(\beta_{\Gamma}\right) & \mu_{\Gamma}-H_{\boldsymbol{k}}^{\frac{3}{2} \oplus \frac{1}{2}}
\end{array}\right) .
$$

Here, $\hat{\Psi}_{\boldsymbol{k}}^{\dagger}=\left(\hat{C}_{\boldsymbol{k}}^{\dagger}, \hat{C}_{-\boldsymbol{k}}^{T} \gamma\right)$ is a spinor with the creation (destruction) operator $\hat{C}_{\boldsymbol{k}}^{\dagger}\left(\hat{C}_{\boldsymbol{k}}\right)$ for electrons of the $H_{\boldsymbol{k}}^{\frac{3}{2} \oplus \frac{1}{2}}$ and $\gamma=\exp \left[-i \pi S_{y}^{(3 / 2)}\right] \oplus \exp \left[-i \pi S_{y}^{(1 / 2)}\right]$ is the unitary part of the time-reversal operator, and $\mu_{\Gamma}$ is the Fermi level measured from the four-fold nodal point.

In the weak pairing limit with $\alpha_{\Gamma}$ much smaller than the energy separation between the bands near the Fermi

level, the BdG spectrum and its topology are largely determined by the intraband gap functions given by

$$
\begin{aligned}
\delta_{\Gamma, \text { in }} & \left.\equiv\left\langle\Gamma, \text { in }, \boldsymbol{k}\left|\Delta_{\Gamma}\left(\beta_{\Gamma}\right)\right| \Gamma, \text { in }, \boldsymbol{k}\right\rangle\right|_{k=k_{\mathrm{F}, \text { in }}} \\
& =\alpha_{\Gamma} \cos \beta_{\Gamma}\left(\tan \beta_{\Gamma}+1\right), \\
\delta_{\Gamma, \text { out }} & \left.\equiv\left\langle\Gamma, \text { out }, \boldsymbol{k}\left|\Delta_{\Gamma}\left(\beta_{\Gamma}\right)\right| \Gamma, \text { out }, \boldsymbol{k} \mid\right\rangle\right|_{k=k_{\mathrm{F}, \text { out }}} \\
& \approx \alpha_{\Gamma} \cos \beta_{\Gamma}\left[\tan \beta_{\Gamma}-g_{\Gamma}\left(\mu_{\Gamma} / m_{\Gamma}\right)\right],
\end{aligned}
$$

with $g_{\Gamma}(x)=\frac{3 x^{2}-2 x-1}{3 x^{2}+2 x+1}$, while the interband gap functions can be treated perturbatively. The approximation in Eq. (8) is valid as long as $\alpha_{\Gamma}$ is sufficiently small compared to $\left|3 \mu_{\Gamma}+m_{\Gamma}\right|$.

The key insight here is that $\mu_{\Gamma}$ determines the sign of $\delta_{\Gamma, \text { out }}$ when $\left|\beta_{\Gamma}\right|<\pi / 4$. This is most apparent in the case of $\beta_{\Gamma}=0$ in Eq. (6) from the wave function character in Eq. (2). Because $\Delta_{\Gamma}(0) \propto \Delta_{ \pm}^{\Gamma}$ in Eq. (6), $\delta_{\Gamma, \text { out }}>0$ for small $\mu_{\Gamma}>0$ since the outer FSS is mainly composed of the RSW fermion. As $\mu_{\Gamma}$ is increased, however, so does the composition of the KW fermion in the outer FSS, and $\Delta_{ \pm}^{\Gamma}$ gives $\delta_{\Gamma, \text { out }}<0$. Hence, the variation in the wave function character switches the sign of $\delta_{\Gamma, \text { out }}$.

In the same manner, we define $\delta_{\mathrm{R} \text {,in }}$ and $\delta_{\mathrm{R} \text {,out }}$, which have the same form as those in Eq. (8) but with $g_{\mathrm{R}}(x)=$ $\frac{2 x-1}{2 x+1}$, and find that the sign of $\delta_{\mathrm{R}, \text { out }}$ also can be flipped by adjusting $\mu_{\mathrm{R}}>0$ when $\left|\beta_{\mathrm{R}}\right|<\pi / 4$.

Phase diagrams in Figs. 2(a) and 2(b) summarize the topological phases at $\Gamma$ and $R$, respectively. When the whole $\mathrm{BdG}$ spectrum is gapped, a winding number $w_{3 \mathrm{D}}$ determines the topology and the number of helical MZMs on the SBZ [19]. However, as explained below, a TSC with nodal rings (NRs) around $\mathrm{R}$ can appear for large $\mu_{\mathrm{R}}$. In this case, the presence/absence of a MZM at $\bar{\Gamma}$ can be determined by a winding number $w_{1 \mathrm{D}}$ [57]. Hence, we use a pair of winding numbers $\left(w_{3 \mathrm{D}}, w_{1 \mathrm{D}}\right)$ to characterize the topology of each region in the phase diagrams.

In the weak pairing limit, $w_{3 \mathrm{D}}$ is expressed as [18]

$$
w_{3 \mathrm{D}}=\frac{1}{2} \sum_{n} \operatorname{sign}\left(\delta_{n}\right) \mathrm{Ch}_{n},
$$

where $\mathrm{Ch}_{n}$ is the Chern number of the $n$th FSS. We break $w_{3 \mathrm{D}}$ into $w_{3 \mathrm{D}, \Gamma}$ and $w_{3 \mathrm{D}, \mathrm{R}}$ according to the centers of FSSs. For $w_{3 \mathrm{D}, \Gamma}$, we expect it to be \pm 2 or +1 , and the latter occurs only when $\delta_{\Gamma, \text { out }} \delta_{\Gamma, \text { in }}<0$. Meanwhile, $w_{3 \mathrm{D}, \mathrm{R}}=-2 \operatorname{sign}\left(\delta_{\mathrm{R}, \text { in }}\right)$ is always even. Consequently, $w_{3 \mathrm{D}}$ is odd whenever $\delta_{\Gamma, \text { out }} \delta_{\Gamma \text {,in }}<0$, and we expect at least one topologically protected MZM at $\bar{\Gamma}$ on SBZ.

Unlike $\delta_{\Gamma, \text { out }}$, the sign flip of $\delta_{\mathrm{R} \text {,out }}$ with $\mu_{\mathrm{R}}$ has no effect on $w_{3 \mathrm{D}}$ since $\mathrm{Ch}_{\mathrm{R} \text {,out }}=0$. Nevertheless, it can lead to a nodal TSC in RhSi for sufficiently large $\mu_{\mathrm{R}}$, around which the band structure can be understood by considering the anisotropic $\boldsymbol{k}$-quadratic correction to $H_{k}^{1 \oplus 0} \oplus H_{k}^{1 \oplus 0}$. Breaking the artificial isotropy of $H_{\boldsymbol{k}}^{1 \oplus 0} \oplus H_{\boldsymbol{k}}^{1 \oplus 0}$ in $\boldsymbol{k}$, it modifies the band structure in 
Fig. 1(c) in two ways. First, there appear band crossings along the $\mathrm{R} \Gamma$ lines because of the symmetry-enforced band connectivity [58]. Second, the double degeneracy of the model in Eq. (3) is lifted in generic momenta in BZ except on $k_{x, y, z}=\pi$ planes due to the local Kramers theorem from the two-fold screw symmetries and the timereversal symmetry [35].

Both effects are clearly shown in Fig. 2(c). The bands crossing the Fermi level $\mu_{\mathrm{R}}>0$ are labeled by $n=1,2,3,4$ in the order of closeness to $\mathrm{R}$, and the color represents the sign of $\delta_{\mathrm{R}, n}$ for $\beta_{\mathrm{R}}=0$ in Eq (6). As explained, the signs of $\delta_{\mathrm{R}, 3}$ and $\delta_{\mathrm{R}, 4}$ are flipped as $\mu_{\mathrm{R}}$ is raised over $E_{c 1}\left(\beta_{\mathrm{R}}\right)=\frac{m_{\mathrm{R}}}{2}\left(\frac{1+\tan \beta_{\mathrm{R}}}{1-\tan \beta_{\mathrm{R}}}\right)$ at which $g_{\mathrm{R}}\left(\mu_{\mathrm{R}} / m_{\mathrm{R}}\right)=\tan \beta_{\mathrm{R}}$. For $\beta_{\mathrm{R}}=0, E_{c 1}=m_{\mathrm{R}} / 2$. The switched signs are maintained for the higher energies.

The BdG spectrum around $\mathrm{R}$ remains gapped until $\mu_{\mathrm{R}}$ reaches the band crossing energy $E_{c 2}$. For $\mu_{\mathrm{R}}>E_{c 2}$, however, topological NRs in the BdG spectrum appear because the interchange of wave functions between band 2 and 3 at the band crossing on the $\mathrm{R} \Gamma$ line makes the signs of $\delta_{\mathrm{R}, 2(3)}$ on the $\mathrm{R} \Gamma$ line and on the $k_{x, y, z}=\pi$ planes opposite. Suppose P (Q) to be a point where the FSS of band 2 and the R $\Gamma$ line (RX line) meet as shown in Fig. 2(d). Since $\delta_{\mathrm{P}} \delta_{\mathrm{Q}}<0$, there exists at least a point where the gap function becomes zero for any path between $\mathrm{P}$ and $\mathrm{Q}$ on the FSS. As a result, a ring with $\delta_{\mathrm{R}, 2}=0$ wrapping the $\mathrm{R} \Gamma$ line appears on the FSS of band 2. For the same reason, another NR is found on the FSS of band 3. The stability of NRs is guaranteed by the $1 \mathrm{D}$ winding number for AIII class evaluated along a loop enclosing the ring [59]. The rings from band 2 and 3 have opposite winding numbers of unit magnitude (see Sec. IV of SM [51]).

Even in the presence of NRs around R, a MZM at $\bar{\Gamma}$ is still protected by $w_{1 \mathrm{D}}$ evaluated along a TRI loop $\mathcal{L}$ connecting $\Gamma$ with another TRIM that is projected to $\bar{\Gamma}$ on the SBZ without intersecting with NRs in the BZ [59]. In the weak pairing limit, $w_{1 \mathrm{D}}$ can be easily evaluated from [18]

$$
w_{1 \mathrm{D}}=\prod_{n \in \mathrm{FSS}^{\prime}} \operatorname{sign}\left(\delta_{n}\right),
$$

where FSS $^{\prime}$ denotes the FSSs which intersect with $\mathcal{L}$. Note that the four FSSs near R do not contribute to $w_{1 \mathrm{D}}$ due to the local Kramers degeneracies implying $\delta_{\mathrm{R}, 2 n-1}=\delta_{\mathrm{R}, 2 n}$ for $n=1,2$. Thus, a MZM at $\bar{\Gamma}$ is expected for any surfaces of the crystal as long as $\delta_{\Gamma, \text { in }} \delta_{\Gamma, \text { out }}<0$, which is consistent with the condition in the fully gapped phase.

TSC phase diagram in various interactions.- To investigate what kind of interactions realize the $s_{+} \oplus s_{-}$gap functions, we consider three types of electron-electron interactions:

$$
\hat{H}_{\mathrm{int}}=\frac{U}{2} \sum_{i} \hat{\rho}_{i} \hat{\rho}_{i}+\frac{V}{2} \sum_{\langle i, j\rangle} \hat{\rho}_{i} \hat{\rho}_{j}+\frac{J}{2} \sum_{\langle i, j\rangle} \hat{\boldsymbol{S}}_{i} \cdot \hat{\boldsymbol{S}}_{j} .
$$
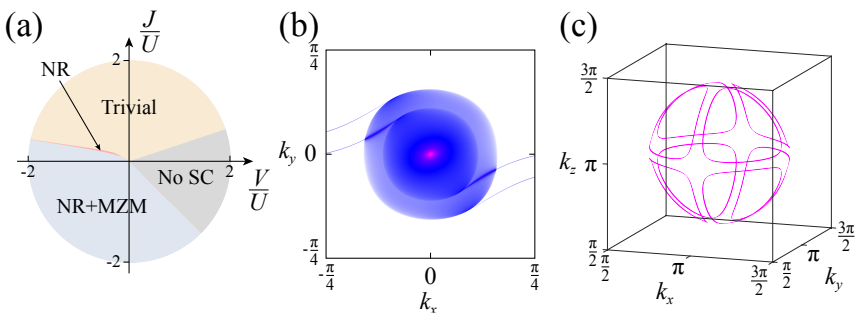

Figure 3. (a) Phase diagram in $(V / U, J / U)$ at $\mu=1.3 m_{\Gamma}$ with $U>0$. "No SC" and "Trivial" mean no superconductivity and the topologically trivial superconducting phase, respectively, whereas "NR" and "MZM" represent the superconducting phases with the NRs around R, and a MZM at $\bar{\Gamma}$, respectively. (b) Surface spectral weight around $\bar{\Gamma}$ at $E=0$. (c) Nodal rings around $\mathrm{R}$ in the BZ.

Here, $\quad\left(\hat{\rho}_{i}, \hat{\boldsymbol{S}}_{i}\right)=\left(\hat{C}_{i, \uparrow}^{\dagger}, \hat{C}_{i, \downarrow}^{\dagger}\right)\left(s_{0}, \boldsymbol{s}\right)\left(\hat{C}_{i, \uparrow}, \hat{C}_{i, \downarrow}\right)^{T}$ with the spin Pauli matrices $s . \quad U, V$ and $J$ represent the renormalized on-site Coulomb interaction, the nearest-neighbor Coulomb interaction, and the nearestneighbor (anti)ferromagnetic exchange interaction, respectively [60]. Considering only the time-reversal invariant pairing channels from $\hat{H}_{\text {int }}$ respecting spatial symmetries of RhSi, the pairing interaction $\hat{H}_{\text {pair }}$ can be expressed as

$$
\hat{H}_{\text {pair }}=\sum_{i=0}^{8} \frac{U_{i}}{4} \sum_{\boldsymbol{k}, \boldsymbol{p}} \hat{\Pi}_{i}(\boldsymbol{k}) \hat{\Pi}_{i}^{\dagger}(\boldsymbol{p}),
$$

where $\hat{\Pi}_{i}(\boldsymbol{k})=\hat{C}_{-\boldsymbol{k}}^{T} \gamma M_{i, \boldsymbol{k}}^{\dagger} \hat{C}_{\boldsymbol{k}}$, and $U_{i}$ 's are the coupling constants of the pairing channels $\hat{\Pi}_{i} \hat{\Pi}_{i}^{\dagger}$ with $U_{0}=U / 4$, $U_{1,5}=V-3 J$, and $U_{2,3,4,6,7,8}=V+J$. Here, $M_{i, \boldsymbol{k}}$ are $\boldsymbol{k}$-dependent matrices characterizing the pairing channels (see Sec. I of SM [51]).

Solving the linearized gap equation with $\hat{H}_{\text {pair }}$, the gap function $\Delta(\boldsymbol{k})=\sum_{i=0}^{8} \Delta_{i} M_{i, \boldsymbol{k}}$ with the highest transition temperature is obtained. The relationship between $\Delta(\boldsymbol{k})$ and Eq. (6) can be understood by projecting $\Delta(\boldsymbol{k})$ to the bases of $H_{\boldsymbol{k}}^{\frac{3}{2} \oplus \frac{1}{2}}$ and $H_{\boldsymbol{k}}^{1 \oplus 0}$ at $\Gamma$ and R, respectively. At $\Gamma, \Delta(\boldsymbol{k}) \sim\left(\Delta_{0}-\Delta_{1}\right) I_{6}+\left(\Delta_{2}-\Delta_{3}\right) \Delta_{ \pm}^{\Gamma}$, whereas at R, $\Delta(\boldsymbol{k}) \sim \Delta_{0} I_{4}+\Delta_{4} \Delta_{ \pm}^{\mathrm{R}}$ up to the zeroth order in $\boldsymbol{k}$. For $U<0$, the trivial gap function largely prevails. Hence, we focus on $U>0$.

Figure $3(\mathrm{a})$ shows the phase diagram in $(V / U, J / U)$ for $U>0$ at $\mu=\mu_{\Gamma}=\mu_{\mathrm{R}}-0.43 \mathrm{eV}=1.3 m_{\Gamma}$. In the blue "NR+MZM" region, we find that the $s_{+} \oplus s_{-}$gap functions are dominant and the resultant TSC exhibits a MZM at $\bar{\Gamma}$ and the NRs near R as shown in Figs. 3(b) and $3(\mathrm{c})$, respectively. This region is approximately covered by $V<-J$ with $J<0$, where the ferromagnetic interaction is stronger than the nearest-neighborhood Coulomb interaction. In a narrow region in red at the boundary of the blue region, the TSC with only the NRs from $\Delta_{ \pm}^{\mathrm{R}}$ appears. The beige region marked by "Trivial" approximately overlaps with $V<3 J$ with $J>0$, where 
the topologically trivial SC arises from the spin-singlet pairings due to the anti-ferromagnetic interaction $J>0$ in contrast to the "NR+MZM" region. Near the phase boundaries, TSC phases with additional NRs around $\Gamma$ or $\mathrm{R}$ may appear due to a complicated combination of various gap functions (see Sec. V of SM [51]).

Discussion.- The $s_{+} \oplus s_{-}$gap functions in our work should be distinguished from the momentum-dependent $s_{ \pm}$gap functions usually discussed in iron-based superconductors [61]. We find that an analogous gap function for our system, corresponding to $\alpha_{\Gamma} \alpha_{\mathrm{R}}<0$ in Eq. (6) $[45,46]$, is not favored by the electron-electron interaction of Eq. (11) (see Sec. V of SM [51]).

Regarding the experimental realization, we want to refer to the report on the multi-gap superconductivity in RhGe [62] whose electronic structure is akin to RhSi. Considering weak ferromagnetism in $\mathrm{RhGe}$, it is expected that the TSC arising from the $s_{+} \oplus s_{-}$gap functions could be realized by varying its chemical compositions [63]. Also, we expect that our result could be applied to the multi-gap superconductor BeAu [64-66] in B20 structure as well as other noncentrosymmetric superconductors such as $\mathrm{Li}_{2} \mathrm{X}_{3} \mathrm{~B}(\mathrm{X}=\mathrm{Pd}, \mathrm{Pt})$ [46], PtSbS [67], and BaPtP [68] supporting coupled multifold fermions.

The TSC from the $s_{+} \oplus s_{-}$gap functions can be investigated by probing the bulk and surface properties, respectively. To discern the gap function from the trivial $s$-wave gap function, magnetic resonance techniques and angle-resolved photoemission spectroscopy can be used to identify the spin state of the gap function and the size of the superconducting gap on each FSS, respectively $[69,70]$. Regarding the response from the surface, drastic anisotropy in response to magnetic fields could be a smoking gun of the MZMs on the surface [71-73].

This work was supported by the National Research Foundation of Korea (NRF) grant funded by the Korea government (MSIT) (No. 2018R1A2B6007837) and Creative-Pioneering Researchers Program through Seoul National University (SNU). C. Y. was supported by NRF of Korea under Grant No. 2016H1A2A1907780 through the Global PhD Fellowship Program. S.B.C. was supported by NRF grants funded by the Korea government (MSIT) (No. 2020R1A2C1007554) and the Ministry of Education (2018R1A6A1A06024977).

* sbchung0@uos.ac.kr

† hmin@snu.ac.kr

[1] J. Alicea, Rep. Prog. Phys. 75, 076501 (2012).

[2] C. Beenakker, Annu. Rev. Condens. Matter Phys. 4, 113 (2013).

[3] T. D. Stanescu and S. Tewari, J. Phys.: Condens. Matter 25, 233201 (2013).

[4] M. Sato and S. Fujimoto, J. Phys. Soc. Japan 85, 072001 (2016).
[5] M. Sato and Y. Ando, Rep. Progr. Phys. 80, 076501 (2017).

[6] N. Read and D. Green, Phys. Rev. B 61, 10267 (2000).

[7] L. Fidkowski, X. Chen, and A. Vishwanath, Phys. Rev. X 3, 041016 (2013).

[8] T. Sanno, S. Miyazaki, T. Mizushima, and S. Fujimoto, Phys. Rev. B 103, 054504 (2021).

[9] Y. Oreg, G. Refael, and F. von Oppen, Phys. Rev. Lett. 105, 177002 (2010).

[10] J. D. Sau, R. M. Lutchyn, S. Tewari, and S. Das Sarma, Phys. Rev. Lett. 104, 040502 (2010).

[11] R. M. Lutchyn, J. D. Sau, and S. Das Sarma, Phys. Rev. Lett. 105, 077001 (2010).

[12] C.-X. Liu, J. D. Sau, T. D. Stanescu, and S. Das Sarma, Phys. Rev. B 96, 075161 (2017).

[13] E. Prada, P. San-Jose, M. W. de Moor, A. Geresdi, E. J. Lee, J. Klinovaja, D. Loss, J. Nygård, R. Aguado, and L. P. Kouwenhoven, Nat. Rev. Phys. 2, 575 (2020).

[14] S. Nadj-Perge, I. K. Drozdov, J. Li, H. Chen, S. Jeon, J. Seo, A. H. MacDonald, B. A. Bernevig, and A. Yazdani, Science 346, 602 (2014).

[15] L. Fu and C. L. Kane, Phys. Rev. Lett. 100, 096407 (2008).

[16] L. Fu and C. L. Kane, Phys. Rev. B 79, 161408(R) (2009).

[17] L. Fu, C. L. Kane, and E. J. Mele, Phys. Rev. Lett. 98, 106803 (2007).

[18] X. L. Qi, T. L. Hughes, and S. C. Zhang, Phys. Rev. B 81, 134508 (2010).

[19] C.-K. Chiu, J. C. Y. Teo, A. P. Schnyder, and S. Ryu, Rev. Mod. Phys. 88, 035005 (2016).

[20] E.-G. Moon, C. Xu, Y. B. Kim, and L. Balents, Phys. Rev. Lett. 111, 206401 (2013).

[21] I. F. Herbut and L. Janssen, Phys. Rev. Lett. 113, 106401 (2014)

[22] J. Maciejko and R. Nandkishore, Phys. Rev. B 90, 035126 (2014).

[23] F. Detassis, L. Fritz, and S. Grubinskas, Phys. Rev. B 96, 195157 (2017).

[24] S. Han, C. Lee, E.-G. Moon, and H. Min, Phys. Rev. Lett. 122, 187601 (2019).

[25] W. Shi, B. J. Wieder, H. L. Meyerheim, Y. Sun, Y. Zhang, Y. Li, L. Shen, Y. Qi, L. Yang, J. Jena, P. Werner, K. Koepernik, S. Parkin, Y. Chen, C. Felser, B. A. Bernevig, and Z. Wang, Nat. Phys. 17, 381 (2021).

[26] X. Wan, A. M. Turner, A. Vishwanath, and S. Y. Savrasov, Phys. Rev. B 83, 205101 (2011).

[27] A. A. Burkov and L. Balents, Phys. Rev. Lett. 107, 127205 (2011).

[28] T. O. Wehling, A. M. Black-Schaffer, and A. V. Balatsky, Adv. Phys. 63, 1 (2014).

[29] N. P. Armitage, E. J. Mele, and A. Vishwanath, Rev. Mod. Phys. 90, 015001 (2018).

[30] S. Murakami, N. Nagaosa, and S.-C. Zhang, Phys. Rev. B 69, 235206 (2004).

[31] T. Kondo, M. Nakayama, R. Chen, J. J. Ishikawa, E. G. Moon, T. Yamamoto, Y. Ota, W. Malaeb, H. Kanai, Y. Nakashima, Y. Ishida, R. Yoshida, H. Yamamoto, M. Matsunami, S. Kimura, N. Inami, K. Ono, H. Kumigashira, S. Nakatsuji, L. Balents, and S. Shin, Nat. Commun. 6, 10042 (2015).

[32] B. Bradlyn, J. Cano, Z. Wang, M. G. Vergniory, C. Felser, R. J. Cava, and B. A. Bernevig, Science 353, aaf5037 (2016). 
[33] S. Mühlbauer, B. Binz, F. Jonietz, C. Pfleiderer, A. Rosch, A. Neubauer, R. Georgii, and P. Böni, Science 323, 915 (2009).

[34] D. A. Pshenay-Severin and A. T. Burkov, Materials 12 (2019).

[35] G. Chang, S.-Y. Xu, B. J. Wieder, D. S. Sanchez, S.-M. Huang, I. Belopolski, T.-R. Chang, S. Zhang, A. Bansil, H. Lin, and M. Z. Hasan, Phys. Rev. Lett. 119, 206401 (2017).

[36] Z. Ni, B. Xu, M. Á. Sánchez-Martínez, Y. Zhang, K. Manna, C. Bernhard, J. W. F. Venderbos, F. de Juan, C. Felser, A. G. Grushin, and L. Wu, npj Quantum Mater. 5, 96 (2020).

[37] D. Rees, K. Manna, B. Lu, T. Morimoto, H. Borrmann, C. Felser, J. E. Moore, D. H. Torchinsky, and J. Orenstein, Sci. Adv. 6, eaba0509 (2020).

[38] D. S. Sanchez, I. Belopolski, T. A. Cochran, X. Xu, J.X. Yin, G. Chang, W. Xie, K. Manna, V. Süß, C.-Y. Huang, N. Alidoust, D. Multer, S. S. Zhang, N. Shumiya, X. Wang, G.-Q. Wang, T.-R. Chang, C. Felser, S.-Y. Xu, S. Jia, H. Lin, and M. Z. Hasan, Nature (London) 567, 500 (2019).

[39] D. Takane, Z. Wang, S. Souma, K. Nakayama, T. Nakamura, H. Oinuma, Y. Nakata, H. Iwasawa, C. Cacho, T. Kim, K. Horiba, H. Kumigashira, T. Takahashi, Y. Ando, and T. Sato, Phys. Rev. Lett. 122, 076402 (2019).

[40] Z. Rao, H. Li, T. Zhang, S. Tian, C. Li, B. Fu, C. Tang, L. Wang, Z. Li, W. Fan, J. Li, Y. Huang, Z. Liu, Y. Long, C. Fang, H. Weng, Y. Shi, H. Lei, Y. Sun, T. Qian, and H. Ding, Nature (London) 567, 496 (2019).

[41] N. B. M. Schröter, D. Pei, M. G. Vergniory, Y. Sun, K. Manna, F. de Juan, J. A. Krieger, V. Süss, M. Schmidt, P. Dudin, B. Bradlyn, T. K. Kim, T. Schmitt, C. Cacho, C. Felser, V. N. Strocov, and Y. Chen, Nat. Phys. 15, 759 (2019).

[42] H. Isobe and L. Fu, Phys. Rev. B 93, 241113(R) (2016).

[43] J. M. Link, I. Boettcher, and I. F. Herbut, Phys. Rev. B 101, 184503 (2020).

[44] I. Boettcher, Phys. Rev. Lett. 124, 127602 (2020).

[45] Y. Huang and S.-K. Jian, Phys. Rev. B 103, L161113 (2021).

[46] J. Z. S. Gao, X.-J. Gao, W.-Y. He, X. Y. Xu, T. K. Ng, and K. T. Law, arXiv:2012.11287.

[47] X. Dai, Z. Fang, Y. Zhou, and F.-C. Zhang, Phys. Rev. Lett. 101, 057008 (2008).

[48] T. Nomoto, K. Hattori, and H. Ikeda, Phys. Rev. B 94, 174513 (2016).

[49] H. G. Suh, H. Menke, P. M. R. Brydon, C. Timm, A. Ramires, and D. F. Agterberg, Phys. Rev. Research 2, 032023(R) (2020).

[50] C. Setty, S. Bhattacharyya, Y. Cao, A. Kreisel, and P. J. Hirschfeld, Nat. Commun. 11, 523 (2020).

[51] See Supplemental Material at http://link.aps.org/ supplemental/10.1103/PhysRevB.104.L241115 for the details of the lattice and continuum models for $\mathrm{RhSi}$, gap functions, generic DIII topological phases with the $s_{+} \oplus s_{-}$gap functions, nodal rings around $\mathrm{R}$ and their stability, and linearized gap equation, which includes Ref. [52].

[52] C. Fang, H. Weng, X. Dai, and Z. Fang, Chin. Phys. B 25, 117106 (2016).

[53] H. Hu, J. Hou, F. Zhang, and C. Zhang, Phys. Rev. Lett. 120, 240401 (2018).

[54] I. C. Fulga and A. Stern, Phys. Rev. B 95, 241116(R) (2017).

[55] Y.-P. Lin and R. M. Nandkishore, Phys. Rev. B 97, 134521 (2018).

[56] H. Nielsen and M. Ninomiya, Nucl. Phys. B 185, 20 (1981).

[57] A. P. Schnyder, P. M. R. Brydon, and C. Timm, Phys. Rev. B 85, 024522 (2012).

[58] D. A. Pshenay-Severin, Y. V. Ivanov, A. A. Burkov, and A. T. Burkov, J. Phys. Condens. Matter 30, 135501 (2018).

[59] A. P. Schnyder and S. Ryu, Phys. Rev. B 84, 060504(R) (2011).

[60] O. Vafek and A. V. Chubukov, Phys. Rev. Lett. 118, 087003 (2017).

[61] A. Chubukov, Annu. Rev. Condens. Matter Phys. 3, 57 (2012).

[62] A. Tsvyashchenko, V. Sidorov, A. Petrova, L. Fomicheva, I. Zibrov, and V. Dmitrienko, J. Alloys Compd. 686, 431 (2016).

[63] D. Salamatin, A. Tsvyashchenko, A. Salamatin, A. Velichkov, M. Magnitskaya, N. Chtchelkatchev, V. Sidorov, L. Fomicheva, M. Mikhin, M. Kozin, A. Nikolaev, I. Romashkina, and M. Budzynski, J. Alloys Compd. 850, 156601 (2021).

[64] B. Matthias, J. Phys. Chem. Solids 10, 342 (1959).

[65] D. J. Rebar, S. M. Birnbaum, J. Singleton, M. Khan, J. C. Ball, P. W. Adams, J. Y. Chan, D. P. Young, D. A. Browne, and J. F. DiTusa, Phys. Rev. B 99, 094517 (2019).

[66] R. Khasanov, R. Gupta, D. Das, A. Amon, A. LeitheJasper, and E. Svanidze, Phys. Rev. Research 2, 023142 (2020).

[67] R. Mizutani, Y. Okamoto, H. Nagaso, Y. Yamakawa, H. Takatsu, H. Kageyama, S. Kittaka, Y. Kono, T. Sakakibara, and K. Takenaka, J. Phys. Soc. Japan 88, 093709 (2019).

[68] Y. Okamoto, R. Mizutani, Y. Yamakawa, H. Takatsu, H. Kageyama, and K. Takenaka, JPS Conf. Proc. 29, 011001 (2020).

[69] K. Ishida, H. Mukuda, Y. Kitaoka, K. Asayama, Z. Mao, Y. Mori, and Y. Maeno, Nature (London) 396, 658 (1998).

[70] D. Mou, R. Jiang, V. Taufour, S. L. Bud'ko, P. C. Canfield, and A. Kaminski, Phys. Rev. B 91, 214519 (2015).

[71] S. B. Chung and S.-C. Zhang, Phys. Rev. Lett. 103, 235301 (2009)

[72] S. B. Chung, J. Horowitz, and X.-L. Qi, Phys. Rev. B 88, 214514 (2013).

[73] L. Chirolli and F. Guinea, Phys. Rev. B 99, 014506 (2019). 


\section{Supplemental Material: Topological Multiband s-wave Superconductivity in Coupled Multifold Fermions}

\section{CONTENTS}

I. Lattice and Continuum Models for RhSi 2

A. Tight-binding model 2

B. Continuum model near the $\Gamma$ point 2

C. Continuum model near the R point 4

II. Gap functions 6

A. Expansion of $M_{i, \boldsymbol{k}}$ near the $\Gamma$ point 7

B. Expansion of $M_{i, \boldsymbol{k}}$ near the R point 7

III. Generic DIII Topological Superconducting phases with the $s_{+} \oplus s_{-}$gap functions 8

IV. Nodal rings around $\mathrm{R}$ and their stability 8

V. Linearized gap equation 10

A. Calculation of the linearized gap equation 10

B. $\Delta_{ \pm}, I_{6(4)}$ and other superconducting gap functions 11

C. Ambiguity of SOC parameters and their effect on the superconducting gap functions 11

References 12 


\section{LATTICE AND CONTINUUM MODELS FOR RHSI}

\section{A. Tight-binding model}

In the main text, we use the tight-binding model proposed in Ref. [1]. The model uses a $s$-wave-like orbital at Wyckoff positions with the minimum multiplicity of 4 , and the corresponding four sites $A=(0,0,0), B=\left(\frac{1}{2}, \frac{1}{2}, 0\right)$, $C=\left(\frac{1}{2}, 0, \frac{1}{2}\right)$ and $D=\left(0, \frac{1}{2}, \frac{1}{2}\right)$ are included in the unit cell. (Here we set the lattice constant to unity.) The resultant normal phase Hamiltonian in the basis $\left(A_{\uparrow}, A_{\downarrow}, C_{\uparrow}, C_{\downarrow}, B_{\uparrow}, B_{\downarrow}, D_{\uparrow}, D_{\downarrow}\right)$ is given by

$$
\begin{aligned}
H_{\mathrm{TB}}(\boldsymbol{k}) & =H_{\mathrm{TB}}^{\mathrm{w} / \mathrm{oSOC}}(\boldsymbol{k})+v_{r, 1} M_{2, \boldsymbol{k}}+v_{r, 2} M_{3, \boldsymbol{k}}+v_{r, 3} M_{4, \boldsymbol{k}}+v_{s, 1} M_{6, \boldsymbol{k}}+v_{s, 2} M_{7, \boldsymbol{k}}+v_{s, 3} M_{8, \boldsymbol{k}} \\
H_{\mathrm{TB}}^{\mathrm{w} / \mathrm{o} \mathrm{SOC}}(\boldsymbol{k}) & =v_{2}\left[\sum_{i=x, y, z} \cos k_{i}-\varepsilon_{\mathrm{offset}}\right] M_{0}+v_{1} M_{1, \boldsymbol{k}}+v_{p} M_{5, \boldsymbol{k}}
\end{aligned}
$$

where $\varepsilon_{\text {offset }}=-v_{1}+3 v_{2}+v_{r, 1}-v_{r, 2}$ is introduced to set the energy level of the RSW nodal point at $\Gamma$ as zero. The nine $M_{i, \boldsymbol{k}}$ matrices are given by

$$
\begin{aligned}
M_{0} & =I_{8}, \\
M_{1, \boldsymbol{k}} & =\tau_{1} \cos \frac{k_{x}}{2} \cos \frac{k_{y}}{2}+\tau_{1} \mu_{1} \cos \frac{k_{y}}{2} \cos \frac{k_{z}}{2}+\mu_{1} \cos \frac{k_{z}}{2} \cos \frac{k_{x}}{2}, \\
M_{2, \boldsymbol{k}} & =\tau_{2} \mu_{3} s_{2} \cos \frac{k_{x}}{2} \cos \frac{k_{y}}{2}+\tau_{2} \mu_{1} s_{3} \cos \frac{k_{y}}{2} \cos \frac{k_{z}}{2}+\mu_{2} s_{1} \cos \frac{k_{z}}{2} \cos \frac{k_{x}}{2}, \\
M_{3, \boldsymbol{k}} & =\tau_{2} s_{3} \cos \frac{k_{x}}{2} \cos \frac{k_{y}}{2}+\tau_{1} \mu_{2} s_{1} \cos \frac{k_{y}}{2} \cos \frac{k_{z}}{2}+\tau_{3} \mu_{2} s_{2} \cos \frac{k_{z}}{2} \cos \frac{k_{x}}{2}, \\
M_{4, \boldsymbol{k}} & =\tau_{2} \mu_{3} s_{1} \sin \frac{k_{x}}{2} \sin \frac{k_{y}}{2}+\tau_{2} \mu_{1} s_{2} \sin \frac{k_{y}}{2} \sin \frac{k_{z}}{2}+\mu_{2} s_{3} \sin \frac{k_{z}}{2} \sin \frac{k_{x}}{2}, \\
M_{5, \boldsymbol{k}} & =\tau_{2} \mu_{3} \cos \frac{k_{x}}{2} \sin \frac{k_{y}}{2}+\tau_{2} \mu_{1} \cos \frac{k_{y}}{2} \sin \frac{k_{z}}{2}+\mu_{2} \cos \frac{k_{z}}{2} \sin \frac{k_{x}}{2}, \\
M_{6, \boldsymbol{k}} & =\tau_{1} s_{1} \sin \frac{k_{x}}{2} \cos \frac{k_{y}}{2}+\tau_{1} \mu_{1} s_{2} \sin \frac{k_{y}}{2} \cos \frac{k_{z}}{2}+\mu_{1} s_{3} \sin \frac{k_{z}}{2} \cos \frac{k_{x}}{2}, \\
M_{7, \boldsymbol{k}} & =\tau_{1} s_{2} \cos \frac{k_{x}}{2} \sin \frac{k_{y}}{2}+\tau_{1} \mu_{1} s_{3} \cos \frac{k_{y}}{2} \sin \frac{k_{z}}{2}+\mu_{1} s_{1} \cos \frac{k_{z}}{2} \sin \frac{k_{x}}{2}, \\
M_{8, \boldsymbol{k}} & =\tau_{1} \mu_{3} s_{3} \cos \frac{k_{x}}{2} \sin \frac{k_{y}}{2}-\tau_{2} \mu_{2} s_{1} \cos \frac{k_{y}}{2} \sin \frac{k_{z}}{2}+\tau_{3} \mu_{1} s_{2} \cos \frac{k_{z}}{2} \sin \frac{k_{x}}{2},
\end{aligned}
$$

where $\tau_{i}$ and $\mu_{i}$ are Pauli matrices related to the orbital degrees of freedom at the four Wyckoff positions $A, B, C$ and $D$, and $s_{i}$ are Pauli matrices operating on the real spin degrees of freedom. $\varepsilon_{\text {offset }}$ is fitted to $-0.04 \mathrm{eV}$. In the main text, we take $v_{1}=0.55, v_{2}=0.16, v_{p}=-0.76, v_{r, 1}=0, v_{r, 2}=-0.03, v_{r, 3}=0.01, v_{s, 1}=0, v_{s, 2}=0$, and $v_{s, 3}=0$ in eV which are different from Ref. [1] where $v_{s, 1}=-0.04$ is used. Considering that $M_{6-8, \boldsymbol{k}}$ are odd functions of $\boldsymbol{k}$ around $\Gamma$ and $\mathrm{R}$, the role of small $v_{s, i}$ 's is to slightly modify the Fermi velocity of the bands which is mainly determined by $v_{p}$. Also, because of the limited usage of atomic basis, the tight-binding model given here does not accurately capture the electronic structure all over the range we are interested in. For these reasons, there is an unavoidable ambiguity in the numerical values for $v_{s, i}$ 's. However, we find that the result we report remains qualitatively unchanged in a reasonable range of $v_{s, i}$ 's, as explained in detail in Sec. V. Hence, we take $v_{s, i}=0$ for simplicity.

\section{B. Continuum model near the $\Gamma$ point}

At $\Gamma, H_{\mathrm{TB}}^{\mathrm{w} / \mathrm{SOC}}(\boldsymbol{k})$ in Eq. (1) yields 6-fold and 2-fold nodal points at the energy level $\varepsilon_{6 \text {-fold }}$ and $\varepsilon_{2 \text {-fold }}$, respectively. The Fermi energy lies near the 6 -fold nodal point for RhSi. As the energy difference $\varepsilon_{2 \text {-fold }}-\varepsilon_{6 \text {-fold }}$ is relatively huge, approximately $2 \mathrm{eV}$ in RhSi, we can obtain the effective $6 \times 6$ Hamiltonian near $\Gamma$ by projecting out the degrees of freedom of the 2-fold nodal point:

$$
H^{(\Gamma)}(\boldsymbol{k})=\mathbb{P}_{\Gamma}^{\dagger} H_{\mathrm{TB}}(\boldsymbol{k}) \mathbb{P}_{\Gamma}
$$


where $\mathbb{P}_{\Gamma}$ is a $8 \times 6$ matrix given by

$$
\mathbb{P}_{\Gamma}=\frac{1}{2}\left(\begin{array}{cccccc}
-z_{1}^{*} & \frac{-2 i}{\sqrt{6}} & \frac{z_{1}}{\sqrt{3}} & 0 & \frac{i}{\sqrt{3}} & \frac{2 z_{1}}{\sqrt{6}} \\
0 & \frac{-z_{1}^{*}}{\sqrt{3}} & \frac{-2 i}{\sqrt{6}} & -z_{1} & \frac{-2 z_{1}^{*}}{\sqrt{6}} & \frac{-i}{\sqrt{3}} \\
z_{1}^{*} & \frac{-2 i}{\sqrt{6}} & \frac{z_{1}}{\sqrt{3}} & 0 & \frac{i}{\sqrt{3}} & \frac{-2 z_{1}}{\sqrt{6}} \\
0 & \frac{z_{1}^{*}}{\sqrt{3}} & \frac{-2 i}{\sqrt{6}} & z_{1} & \frac{2 z_{1}^{*}}{\sqrt{6}} & \frac{-i}{\sqrt{3}} \\
z_{1} & \frac{2 i}{\sqrt{6}} & \frac{z_{1}^{*}}{\sqrt{3}} & 0 & \frac{-i}{\sqrt{3}} & \frac{-2 z_{1}^{*}}{\sqrt{6}} \\
0 & \frac{z_{1}}{\sqrt{3}} & \frac{2 i}{\sqrt{6}} & z_{1}^{*} & \frac{2 z_{1}}{\sqrt{6}} & \frac{i}{\sqrt{3}} \\
-z_{1} & \frac{2 i}{\sqrt{6}} & \frac{-z_{1}^{*}}{\sqrt{3}} & 0 & \frac{-i}{\sqrt{3}} & \frac{2 z_{1}^{*}}{\sqrt{6}} \\
0 & \frac{-z_{1}}{\sqrt{3}} & \frac{2 i}{\sqrt{6}} & -z_{1}^{*} & \frac{-2 z_{1}}{\sqrt{6}} & \frac{i}{\sqrt{3}}
\end{array}\right)
$$

with $z_{1}=e^{\frac{\pi i}{4}} . \mathbb{P}_{\Gamma}$ is constructed from the eigenvectors of $H_{\mathrm{TB}}^{\mathrm{w} / \mathrm{OSOC}}(\mathbf{0})$ satisfying $H_{\mathrm{TB}}^{\mathrm{w} / \mathrm{o} \mathrm{SOC}}(\mathbf{0}) \mathbb{P}_{\Gamma}=\varepsilon_{6 \text {-fold }} \mathbb{P}_{\Gamma}$. Expanding $H^{(\Gamma)}(\boldsymbol{k})$ to linear order in $\boldsymbol{k}$, we have

$$
\begin{aligned}
H^{(\Gamma)}(\boldsymbol{k}) & =H_{\boldsymbol{k}}^{\frac{3}{2} \oplus \frac{1}{2}}+\sum_{i=1,2,3} v_{s, i} \delta H_{s, i}^{(\Gamma)}(\boldsymbol{k})+O\left(k^{2}\right), \\
H_{\boldsymbol{k}}^{\frac{3}{2} \oplus \frac{1}{2}} & =\left(\begin{array}{cc}
v_{\Gamma} \boldsymbol{k} \cdot \boldsymbol{S}^{\left(\frac{3}{2}\right)} & T_{\Gamma, \boldsymbol{k}} \\
T_{\Gamma, \boldsymbol{k}}^{\dagger} & 2 v_{\Gamma} \boldsymbol{k} \cdot \boldsymbol{S}^{\left(\frac{1}{2}\right)}-m_{\Gamma}
\end{array}\right),
\end{aligned}
$$

where $m_{\Gamma}=3 v_{r, 1}-3 v_{r, 2}, v_{\Gamma}=v_{p} / 3$ and

$$
T_{\Gamma, k}^{\dagger}=\frac{v_{\Gamma}}{2 \sqrt{2}}\left(\begin{array}{cccc}
-\sqrt{3} k_{+} & 2 k_{z} & k_{-} & 0 \\
0 & -k_{+} & 2 k_{z} & \sqrt{3} k_{-}
\end{array}\right)
$$

with $k_{ \pm}=k_{x} \pm i k_{y}$. Here, $\delta H_{s, i}^{(\Gamma)}$ is the matrix $M_{5+i, \boldsymbol{k}}$ expanded to the linear order in $\boldsymbol{k}$ :

$$
\begin{aligned}
& \delta H_{s, 1}^{(\Gamma)}(\boldsymbol{k})=\left(\begin{array}{cccccc}
-\frac{k_{z}}{2} & 0 & 0 & -\frac{k_{x}+i k_{y}}{2} & 0 & 0 \\
0 & \frac{k_{z}}{2} & -\frac{k_{x}-i k_{y}}{2} & 0 & 0 & 0 \\
0 & -\frac{k_{x}+i k_{y}}{2} & -\frac{k_{z}}{2} & 0 & 0 & 0 \\
-\frac{k_{x}-i k_{y}}{2} & 0 & 0 & \frac{k_{z}}{2} & 0 & 0 \\
0 & 0 & 0 & 0 & \frac{k_{z}}{2} & \frac{k_{x}-i k_{y}}{2} \\
0 & 0 & 0 & 0 & \frac{k_{x}+i k_{y}}{2} & -\frac{k_{z}}{2}
\end{array}\right), \\
& \delta H_{s, 2}^{(\Gamma)}(\boldsymbol{k})=\left(\begin{array}{cccccc}
0 & -\frac{k_{x}}{2 \sqrt{3}} & \frac{k_{z}}{2 \sqrt{3}} & \frac{i k_{y}}{2} & -\frac{k_{x}}{\sqrt{6}} & -\frac{k_{z}}{\sqrt{6}} \\
-\frac{k_{x}}{2 \sqrt{3}} & -\frac{k_{z}}{3} & \frac{2 k_{x}+i k_{y}}{6} & -\frac{k_{z}}{2 \sqrt{3}} & \frac{k_{z}}{3 \sqrt{2}} & \frac{k_{x}+2 i k_{y}}{3 \sqrt{2}} \\
\frac{k_{z}}{2 \sqrt{3}} & \frac{2 k_{x}-i k_{y}}{6} & \frac{k_{z}}{3} & -\frac{k_{x}}{2 \sqrt{3}} & -\frac{k_{x}-2 i k_{y}}{3 \sqrt{2}} & \frac{k_{z}}{3 \sqrt{2}} \\
-\frac{i k_{y}}{2} & -\frac{k_{z}}{2 \sqrt{3}} & -\frac{k_{x}}{2 \sqrt{3}} & 0 & -\frac{k_{z}}{\sqrt{6}} & \frac{k_{x}}{\sqrt{6}} \\
-\frac{k_{x}}{\sqrt{6}} & \frac{k_{z}}{3 \sqrt{2}} & -\frac{k_{x}+2 i k_{y}}{3 \sqrt{2}} & -\frac{k_{z}}{\sqrt{6}} & -\frac{k_{z}}{6} & -\frac{k_{x}-i k_{y}}{6} \\
-\frac{k_{z}}{\sqrt{6}} & \frac{k_{x}-2 i k_{y}}{3 \sqrt{2}} & \frac{k_{z}}{3 \sqrt{2}} & \frac{k_{x}}{\sqrt{6}} & -\frac{k_{x}+i k_{y}}{6} & \frac{k_{z}}{6}
\end{array}\right), \\
& \delta H_{s, 3}^{(\Gamma)}(\boldsymbol{k})=\left(\begin{array}{cccccc}
0 & -\frac{i k_{y}}{2 \sqrt{3}} & \frac{k_{z}}{2 \sqrt{3}} & -\frac{k_{x}}{2} & \frac{i k_{y}}{2 \sqrt{6}} & \frac{k_{z}}{2 \sqrt{6}} \\
\frac{i k_{y}}{2 \sqrt{3}} & \frac{k_{z}}{3} & \frac{k_{x}+2 i k_{y}}{6} & -\frac{k_{z}}{2 \sqrt{3}} & \frac{k_{z}}{6 \sqrt{2}} & -\frac{2 k_{x}+i k_{y}}{6 \sqrt{2}} \\
\frac{k_{z}}{2 \sqrt{3}} & \frac{k_{x}-2 i k_{y}}{6} & -\frac{k_{z}}{3} & -\frac{i k_{y}}{2 \sqrt{3}} & \frac{2 k_{x}-i k_{y}}{6 \sqrt{2}} & \frac{k_{z}}{6 \sqrt{2}} \\
-\frac{k_{x}}{2} & -\frac{k_{z}}{2 \sqrt{3}} & \frac{i k_{y}}{2 \sqrt{3}} & 0 & \frac{k_{z}}{2 \sqrt{6}} & \frac{i k_{y}}{2 \sqrt{6}} \\
-\frac{i k_{y}}{2 \sqrt{6}} & \frac{k_{z}}{6 \sqrt{2}} & \frac{2 k_{x}+i k_{y}}{6 \sqrt{2}} & \frac{k_{z}}{2 \sqrt{6}} & -\frac{k_{z}}{3} & -\frac{k_{x}-i k_{y}}{3} \\
\frac{k_{z}}{2 \sqrt{6}} & \frac{i k_{y}-2 k_{x}}{6 \sqrt{2}} & \frac{k_{z}}{6 \sqrt{2}} & -\frac{i k_{y}}{2 \sqrt{6}} & -\frac{k_{x}+i k_{y}}{3} & \frac{k_{z}}{3}
\end{array}\right) .
\end{aligned}
$$

As long as $v_{s, i}$ 's are much small in magnitude compared to $v_{p}$, the role of $\delta H_{s, 1-3}$ is just to introduce small anisotropy in the shape of the Fermi surface given by $H^{(\Gamma)}(\boldsymbol{k})$. Thus, for $\left|v_{s, i}\right| \ll\left|v_{p}\right|$, we can neglect them to a good approximation, and thus, $H^{(\Gamma)}(\boldsymbol{k})$ can be approximated as $H_{\boldsymbol{k}}^{\frac{3}{2} \oplus \frac{1}{2}}$ which we use in the main text. 


\section{Continuum model near the $\mathrm{R}$ point}

At R, 6-fold and 2-fold nodal points are obtained from $H_{\mathrm{TB}}\left(\boldsymbol{k}_{\mathrm{R}}\right)$ with $\boldsymbol{k}_{\mathrm{R}}=(\pi, \pi, \pi)$. The matrix of eigenvectors for $H_{\mathrm{TB}}\left(\boldsymbol{k}_{\mathrm{R}}\right)$ is given by

$$
\mathbb{P}_{\mathrm{R}}=\frac{e^{-i \frac{\pi}{4}}}{2}\left(\begin{array}{cccc|cccc}
1 & \frac{i}{\sqrt{2}} & 0 & \frac{i}{\sqrt{2}} & -1 & \frac{i}{\sqrt{2}} & 0 & \frac{i}{\sqrt{2}} \\
0 & \frac{1}{\sqrt{2}} & i & -\frac{1}{\sqrt{2}} & 0 & -\frac{1}{\sqrt{2}} & i & \frac{1}{\sqrt{2}} \\
i & \frac{1}{\sqrt{2}} & 0 & \frac{1}{\sqrt{2}} & -i & \frac{1}{\sqrt{2}} & 0 & \frac{1}{\sqrt{2}} \\
0 & \frac{i}{\sqrt{2}} & 1 & -\frac{i}{\sqrt{2}} & 0 & -\frac{i}{\sqrt{2}} & 1 & \frac{i}{\sqrt{2}} \\
-1 & \frac{i}{\sqrt{2}} & 0 & \frac{i}{\sqrt{2}} & -1 & -\frac{i}{\sqrt{2}} & 0 & -\frac{i}{\sqrt{2}} \\
0 & -\frac{1}{\sqrt{2}} & i & \frac{1}{\sqrt{2}} & 0 & -\frac{1}{\sqrt{2}} & -i & \frac{1}{\sqrt{2}} \\
-i & \frac{1}{\sqrt{2}} & 0 & \frac{1}{\sqrt{2}} & -i & -\frac{1}{\sqrt{2}} & 0 & -\frac{1}{\sqrt{2}} \\
0 & -\frac{i}{\sqrt{2}} & 1 & \frac{i}{\sqrt{2}} & 0 & -\frac{i}{\sqrt{2}} & -1 & \frac{i}{\sqrt{2}}
\end{array}\right) .
$$

Here, the 1, 2, 3, 5, 6, 7-th columns and the 4, 8-th columns of $\mathbb{P}_{\mathrm{R}}$ represent the eigenvectors for the 6-fold and 2-fold nodal points, respectively. In contrast to the case at $\Gamma$, these nodal points are not energetically distant, and hence, the whole 8 degrees of freedom should be considered on equal footing without projecting out some of them. The effective Hamiltonian for electrons near $\mathrm{R}$ is obtained by expanding $H_{\mathrm{TB}}\left(\boldsymbol{k}_{\mathrm{R}}+\boldsymbol{k}\right)$ as

$$
H^{(\mathrm{R})}(\boldsymbol{k})=\mathbb{P}_{\mathrm{R}}^{\dagger} H_{\mathrm{TB}}\left(\boldsymbol{k}_{\mathrm{R}}+\boldsymbol{k}\right) \mathbb{P}_{\mathrm{R}}
$$

To linear order in $\boldsymbol{k}$, we have

$$
\begin{aligned}
H^{(\mathrm{R})}(\boldsymbol{k}) & =\varepsilon_{\mathrm{R}} I_{8}+H_{\boldsymbol{k}}^{1 \oplus 0} \oplus H_{\boldsymbol{k}}^{1 \oplus 0}+\sum_{i=1,2,3} v_{s, i} \delta H_{s, i, \boldsymbol{k}}^{(\mathrm{R})}+O\left(k^{2}\right), \\
H_{\boldsymbol{k}}^{1 \oplus 0} & =\left(\begin{array}{cc}
-v_{\mathrm{R}} \boldsymbol{k} \cdot \boldsymbol{S}^{(1)} & T_{\mathrm{R}, \boldsymbol{k}} \\
T_{\mathrm{R}, \boldsymbol{k}}^{\dagger} & -m_{\mathrm{R}}
\end{array}\right),
\end{aligned}
$$

where $\varepsilon_{\mathrm{R}}=3 v_{2}+v_{r, 3}-\varepsilon_{\text {offset }}, v_{\mathrm{R}}=-v_{p} / 2, m_{\mathrm{R}}=-4 v_{r, 3}$ and

$$
T_{\mathrm{R}, k}^{\dagger}=v_{\mathrm{R}}\left(\begin{array}{lll}
\frac{k_{+}}{\sqrt{2}} & -k_{z} & \frac{-k_{-}}{\sqrt{2}}
\end{array}\right)
$$

$\delta H_{s, i}^{(\mathrm{R})}$ is the matrix $M_{5+i, \boldsymbol{k}_{\mathrm{R}}+\boldsymbol{k}}$ expanded to the linear order in $\boldsymbol{k}$ :

$$
\delta H_{s, 1}^{(\mathrm{R})}(\boldsymbol{k})=\frac{1}{2 \sqrt{2}}\left(\begin{array}{cccccccc}
0 & k_{y} & 0 & -k_{y} & 0 & -k_{x} & -i \sqrt{2} k_{z} & -k_{x} \\
k_{y} & 0 & -k_{y} & 0 & k_{x} & 0 & k_{x} & i \sqrt{2} k_{z} \\
0 & -k_{y} & 0 & -k_{y} & i \sqrt{2} k_{z} & -k_{x} & 0 & k_{x} \\
-k_{y} & 0 & -k_{y} & 0 & k_{x} & -i \sqrt{2} k_{z} & -k_{x} & 0 \\
0 & k_{x} & -i \sqrt{2} k_{z} & k_{x} & 0 & -k_{y} & 0 & k_{y} \\
-k_{x} & 0 & -k_{x} & i \sqrt{2} k_{z} & -k_{y} & 0 & k_{y} & 0 \\
i \sqrt{2} k_{z} & k_{x} & 0 & -k_{x} & 0 & k_{y} & 0 & k_{y} \\
-k_{x} & -i \sqrt{2} k_{z} & k_{x} & 0 & k_{y} & 0 & k_{y} & 0
\end{array}\right),
$$




$$
\delta H_{s, 3}^{(\mathrm{R})}(\boldsymbol{k})=\frac{i}{2 \sqrt{2}}\left(\begin{array}{cccccccc}
0 & 0 & \sqrt{2} k_{z} & 0 & 0 & k_{x}+k_{y} & 0 & k_{x}-k_{y} \\
0 & 0 & 0 & -\sqrt{2} k_{z} & -k_{x}+k_{y} & 0 & -k_{x}-k_{y} & 0 \\
-\sqrt{2} k_{z} & 0 & 0 & 0 & 0 & k_{x}-k_{y} & 0 & -k_{x}-k_{y} \\
0 & \sqrt{2} k_{z} & 0 & 0 & -k_{x}-k_{y} & 0 & k_{x}-k_{y} & 0 \\
0 & k_{x}-k_{y} & 0 & k_{x}+k_{y} & 0 & 0 & 0 & 0 \\
-k_{x}-k_{y} & 0 & -k_{x}+k_{y} & 0 & 0 & 0 & -\sqrt{2} k_{z} & 0 \\
0 & k_{x}+k_{y} & 0 & -k_{x}+k_{y} & \sqrt{2} k_{z} & 0 & 0 & \sqrt{2} k_{z} \\
-k_{x}+k_{y} & 0 & k_{x}+k_{y} & 0 & 0 & -\sqrt{2} k_{z} & 0 & 0
\end{array}\right) .
$$

For $\left|v_{s, i}\right| \ll\left|v_{p}\right|$, we neglect the terms proportional to $v_{s, i}$ in Eq. (14) and we have $H^{(\mathrm{R})}(\boldsymbol{k}) \approx \varepsilon_{\mathrm{R}} I_{8}+H_{\boldsymbol{k}}^{1 \oplus 0} \oplus H_{\boldsymbol{k}}^{1 \oplus 0}$ to linear order in $\boldsymbol{k}$. Since the size of the Fermi sheets around R is large in RhSi, the terms of higher order in $\boldsymbol{k}$ are important to describe the electronic structure near the Fermi energy. Most importantly, the inclusion of quadratic terms in $\boldsymbol{k}$ results in the band crossing as stated in the main text. Written explicitly, the $\boldsymbol{k}$-quadratic correction to $H^{(\mathrm{R})}(\boldsymbol{k})$ is

$$
\delta H^{(2)}(\boldsymbol{k}) \equiv-\frac{v_{2} k^{2}}{2}+v_{1} \delta H_{1, \boldsymbol{k}}^{(\mathrm{R})}+\sum_{i=1,2,3} v_{r, i} \delta H_{r, i, \boldsymbol{k}}^{(\mathrm{R})}
$$

where

$$
\begin{aligned}
& \delta H_{1, \boldsymbol{k}}^{\mathrm{R})}=\frac{1}{4}\left(\begin{array}{cccccccc}
-k_{x} k_{y} & 0 & 0 & 0 & 0 & \frac{k_{z}\left(k_{x}-k_{y}\right)}{\sqrt{2}} & 0 & \frac{k_{z}\left(k_{x}-k_{y}\right)}{\sqrt{2}} \\
0 & 0 & 0 & k_{x} k_{y} & -\frac{k_{z}\left(k_{x}+k_{y}\right)}{\sqrt{2}} & 0 & \frac{k_{z}\left(k_{x}-k_{y}\right)}{\sqrt{2}} & 0 \\
0 & 0 & k_{x} k_{y} & 0 & 0 & -\frac{k_{z}\left(k_{x}+k_{y}\right)}{\sqrt{2}} & 0 & \frac{k_{z}\left(k_{x}+k_{y}\right)}{\sqrt{2}} \\
0 & k_{x} k_{y} & 0 & 0 & -\frac{k_{z}\left(k_{x}+k_{y}\right)}{\sqrt{2}} & 0 & -\frac{k_{z}\left(k_{x}-k_{y}\right)}{\sqrt{2}} & 0 \\
0 & -\frac{k_{z}\left(k_{x}+k_{y}\right)}{\sqrt{2}} & 0 & -\frac{k_{z}\left(k_{x}+k_{y}\right)}{\sqrt{2}} & k_{x} k_{y} & 0 & 0 & 0 \\
\frac{k_{z}\left(k_{x}-k_{y}\right)}{\sqrt{2}} & 0 & -\frac{k_{z}\left(k_{x}+k_{y}\right)}{\sqrt{2}} & 0 & 0 & 0 & 0 & -k_{x} k_{y} \\
0 & \frac{k_{z}\left(k_{x}-k_{y}\right)}{\sqrt{2}} & 0 & -\frac{k_{z}\left(k_{x}-k_{y}\right)}{\sqrt{2}} & 0 & 0 & -k_{x} k_{y} & 0 \\
\frac{k_{z}\left(k_{x}-k_{y}\right)}{\sqrt{2}} & 0 & \frac{k_{z}\left(k_{x}+k_{y}\right)}{\sqrt{2}} & 0 & 0 & -k_{x} k_{y} & 0 & 0
\end{array}\right), \\
& \delta H_{r, 1, \boldsymbol{k}}^{(\mathrm{R})}=\frac{1}{4 \sqrt{2}}\left(\begin{array}{cccc}
0 & k_{z} k_{-} & -i k_{x} k_{y} & -k_{z} k_{+} \\
k_{z} k_{+} & 0 & -k_{z} k_{-} & i k_{x} k_{y} \\
i k_{x} k_{y} & -k_{z} k_{+} & 0 & -k_{z} k_{-} \\
-k_{z} k_{-} & -i k_{x} k_{y} & -k_{z} k_{+} & 0
\end{array}\right) \oplus\left(\begin{array}{cccc}
0 & k_{z} k_{-} & -i k_{x} k_{y} & -k_{z} k_{+} \\
k_{z} k_{+} & 0 & -k_{z} k_{-} & i k_{x} k_{y} \\
i k_{x} k_{y} & -k_{z} k_{+} & 0 & -k_{z} k_{-} \\
-k_{z} k_{-} & -i k_{x} k_{y} & -k_{z} k_{+} & 0
\end{array}\right), \\
& \delta H_{r, 2, k}^{(\mathrm{R})}=\frac{1}{4 \sqrt{2}}\left(\begin{array}{cccccccc}
0 & -k_{y} k_{z} & 0 & k_{y} k_{z} & \sqrt{2} i k_{x} k_{y} & i k_{x} k_{z} & 0 & -i k_{x} k_{z} \\
-k_{y} k_{z} & 0 & -k_{y} k_{z} & 0 & -i k_{x} k_{z} & 0 & i k_{x} k_{z} & \sqrt{2} i k_{x} k_{y} \\
0 & -k_{y} k_{z} & 0 & -k_{y} k_{z} & 0 & -i k_{x} k_{z} & \left.-\sqrt{2} i k_{x} k\right) y & -i k_{x} k_{z} \\
k_{y} k_{z} & 0 & -k_{y} k_{z} & 0 & i k_{x} k_{z} & \sqrt{2} i k_{x} k_{y} & i k_{x} k_{z} & 0 \\
-\sqrt{2} i k_{x} k_{y} & i k_{x} k_{z} & 0 & -i k_{x} k_{z} & 0 & k_{y} k_{z} & 0 & -k_{y} k_{z} \\
-i k_{x} k_{z} & 0 & i k_{x} k_{z} & \sqrt{2} i k_{x} k_{y} & k_{y} k_{z} & 0 & k_{y} k_{z} & 0 \\
0 & -i k_{x} k_{z} & \left.-\sqrt{2} i k_{x} k\right) y & -i k_{x} k_{z} & 0 & -k_{y} k_{z} & 0 & k_{y} k_{z} \\
i k_{x} k_{z} & \sqrt{2} i k_{x} k_{y} & i k_{x} k_{z} & 0 & -k_{y} k_{z} & 0 & k_{y} k_{z} & 0
\end{array}\right), \\
& \delta H_{r, 3, \boldsymbol{k}}^{(\mathrm{R})}=\frac{1}{8}\left(\begin{array}{cccc}
-k_{x}^{2}-k_{z}^{2} & 0 & -k_{x}^{2}+k_{z}^{2} & 0 \\
0 & -2 k_{y}^{2} & 0 & 0 \\
-k_{x}^{2}+k_{z}^{2} & 0 & -k_{x}^{2}-k_{z}^{2} & 0 \\
0 & 0 & 0 & 2\left(k_{x}^{2}+k_{y}^{2}+k_{z}^{2}\right)
\end{array}\right) \oplus\left(\begin{array}{cccc}
-k_{x}^{2}-k_{z}^{2} & 0 & -k_{x}^{2}+k_{z}^{2} & 0 \\
0 & -2 k_{y}^{2} & 0 & 0 \\
-k_{x}^{2}+k_{z}^{2} & 0 & -k_{x}^{2}-k_{z}^{2} & 0 \\
0 & 0 & 0 & 2\left(k_{x}^{2}+k_{y}^{2}+k_{z}^{2}\right)
\end{array}\right) \text {. }
\end{aligned}
$$

Note that only $\delta H_{1, \boldsymbol{k}}^{(\mathrm{R})}$ and $\delta H_{r, 2, \boldsymbol{k}}^{(\mathrm{R})}$ lift the artificial degeneracy in the model $H_{\boldsymbol{k}}^{1 \oplus 0} \oplus H_{\boldsymbol{k}}^{1 \oplus 0}$. Given $v_{1} \gg\left|v_{r, i}\right|$, it is sufficient to keep only $v_{1}$ and $v_{2}$ while neglecting $v_{r, i}$ 's to capture not only the qualitative but also quantitative aspects of the band structure around R. In Fig. S1, the band structures from several continuum models are shown in comparison to the band structure from the tight-binding model. Comparing Figs. S1(a) and S1(b), no significant modification from neglecting $v_{r, i}$ occurs, and especially the location of the band crossing point is well predicted 
even without $v_{r, i}$ 's. Note that the $\boldsymbol{k}$-linear $H_{\boldsymbol{k}}^{1 \oplus 0} \oplus H_{\boldsymbol{k}}^{1 \oplus 0}$ alone does not capture the band crossing near $\mathrm{R}$, as shown in Fig. S1(c). Hence, we conclude that $-v_{2} k^{2} / 2-v_{1} \delta H_{1, \boldsymbol{k}}^{(\mathrm{R})}$ is the major $\boldsymbol{k}$-quadratic correction to $H^{(\mathrm{R})}(\boldsymbol{k}) \approx$ $\varepsilon_{\mathrm{R}} I_{8}+H_{\boldsymbol{k}}^{1 \oplus 0} \oplus H_{\boldsymbol{k}}^{1 \oplus 0}$.
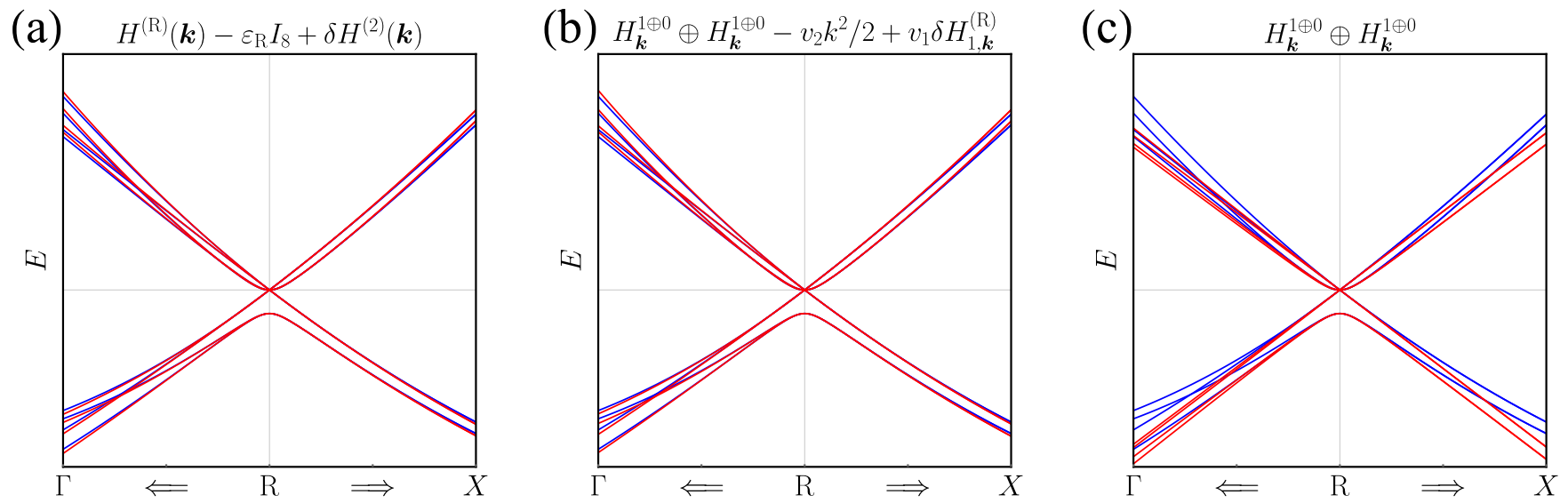

Figure S1: Comparison between the tight-binding band structure (blue line) and the continuum band structure (red line) from (a) $H^{(\mathrm{R})}(\boldsymbol{k})-\varepsilon_{\mathrm{R}}+\delta H^{(2)}(\boldsymbol{k})$ including all $\boldsymbol{k}$-quadratic terms, (b) $H_{\boldsymbol{k}}^{1 \oplus 0} \oplus H_{\boldsymbol{k}}^{1 \oplus 0}-\frac{v_{2} k^{2}}{2}+v_{1} \delta H_{1, \boldsymbol{k}}^{(\mathrm{R})}$, and (c) $H_{\boldsymbol{k}}^{1 \oplus 0} \oplus H_{\boldsymbol{k}}^{1 \oplus 0}$.

\section{GAP FUNCTIONS}

In the main text, we consider only the short-range interactions which result in the short-range Cooper pairings: the pairings between electrons at the same site or the pairings between electrons at the neighboring sites. Focusing on the time-reversal invariant gap functions respecting all the spatial symmetries of $\mathrm{RhSi}$, the gap function $\Delta(\boldsymbol{k})$ can be represented by a linear combination of $M_{i, \boldsymbol{k}}$ 's with $i=0,1,2, \cdots, 8$ :

$$
\Delta(\boldsymbol{k})=\sum_{i=0}^{8} \Delta_{i} M_{i, \boldsymbol{k}}
$$

The basis of the representation is same with that used in Eq. (7) in the main text. Among the 9 matrices, $M_{0, \boldsymbol{k}}$, $M_{1, \boldsymbol{k}}$, and $M_{5, \boldsymbol{k}}$ are the matrices independent of the real spin, and thus represent the real-spin-singlet pairings, while the other 6 matrices denote the real-spin-triplet pairings. Considering the symmetric/antisymmetric character of orbitals in addition to even/odd parity in $\boldsymbol{k}$ and singlet/triplet in spin degrees of freedom, symmetry transformation properties of $M_{i, \boldsymbol{k}}$ are summarized in Table. I. Note that $M_{2, \boldsymbol{k}}, M_{3, \boldsymbol{k}}$, and $M_{4, \boldsymbol{k}}$ are all even-parity, spin-triplet, and orbital-antisymmetric gap functions which correspond to the $s_{+} \oplus s_{-}$gap functions as shown in the following subsections.

\begin{tabular}{|c|c|c|c|c|}
\hline & $\boldsymbol{k}$ & Spin & Orbital & Examples \\
\hline \hline Type 1 & even & singlet & symmetric & $M_{0}, M_{1}$ \\
\hline Type 2 & even & triplet & antisymmetric & $M_{2}, M_{3}, M_{4}$ \\
\hline Type 3 & odd & singlet & symmetric & $M_{5}$ \\
\hline Type 4 & odd & triplet & antisymmetric & $M_{6}, M_{7}, M_{8}$ \\
\hline
\end{tabular}

Table I: Symmetry transformation properties of momentum $(\boldsymbol{k})$, orbital $\left(\tau_{i} \mu_{j}\right)$, and spin $\left(s_{i}\right)$ degrees of freedom under the fermion exchange. For the expression of $M_{i}$ by Pauli matrices $\tau_{i}, \mu_{i}$ and $s_{i}$, see Eq. (3). 


\section{A. Expansion of $M_{i, k}$ near the $\Gamma$ point}

Writing down the first non-zero term, the Taylor expansion of $M_{i, \boldsymbol{k}}^{(\Gamma)} \equiv \mathbb{P}_{\Gamma}^{\dagger} M_{i, \boldsymbol{k}} \mathbb{P}_{\Gamma}$ around $\Gamma$ in $\boldsymbol{k}$ is given by

$$
\begin{array}{lll}
M_{0, \boldsymbol{k}}^{(\Gamma)}=+I_{6}, & M_{1, \boldsymbol{k}}^{(\Gamma)}=-I_{6}, & M_{2, \boldsymbol{k}}^{(\Gamma)}=\Delta_{ \pm}^{\Gamma}, \quad M_{3, \boldsymbol{k}}^{(\Gamma)}=-\Delta_{ \pm}^{\Gamma} \\
M_{6, \boldsymbol{k}}^{(\Gamma)}=\delta H_{s, 1}^{(\Gamma)}(\boldsymbol{k}), & M_{7, \boldsymbol{k}}^{(\Gamma)}=\delta H_{s, 2}^{(\Gamma)}(\boldsymbol{k}), & M_{8, \boldsymbol{k}}^{(\Gamma)}=\delta H_{s, 3}^{(\Gamma)}(\boldsymbol{k}),
\end{array}
$$

and

$$
\begin{aligned}
M_{4, k}^{(\Gamma)}=\left(\begin{array}{cccccc}
0 & \frac{\left(k_{x}-i k_{y}\right) k_{z}}{4 \sqrt{3}} & -\frac{i k_{x} k_{y}}{4 \sqrt{3}} & 0 & -\frac{\left(k_{x}+2 i k_{y}\right) k_{z}}{4 \sqrt{6}} & -\frac{i k_{x} k_{y}}{4 \sqrt{6}} \\
\frac{\left(k_{x}+i k_{y}\right) k_{z}}{4 \sqrt{3}} & 0 & 0 & -\frac{i k_{x} k_{y}}{4 \sqrt{3}} & \frac{i k_{x} k_{y}}{4 \sqrt{2}} & -\frac{k_{x} k_{z}}{4 \sqrt{2}} \\
\frac{i k_{x} k_{y}}{4 \sqrt{3}} & 0 & 0 & -\frac{\left(k_{x}-i k y\right) k_{z}}{4 \sqrt{3}} & -\frac{k_{x} k_{z}}{4 \sqrt{2}} & \frac{i i_{x} k_{y}}{4 \sqrt{2}} \\
0 & \frac{i k_{x} k_{y}}{4 \sqrt{3}} & -\frac{\left(k_{x}+i k_{y}\right) k_{z}}{4 \sqrt{3}} & 0 & -\frac{i k_{x} k_{y}}{4 \sqrt{6}} & -\frac{\left(k_{x}-2 k_{y}\right) k_{z}}{4 \sqrt{6}} \\
-\frac{\left(k_{x}-2 i k_{y}\right) k_{z}}{4 \sqrt{6}} & -\frac{i k_{x} k_{y}}{4 \sqrt{2}} & -\frac{k_{x} k_{z}}{4 \sqrt{2}} & \frac{i k_{x} k_{y}}{4 \sqrt{6}} & 0 & 0 \\
\frac{i k_{x} k_{y}}{4 \sqrt{6}} & -\frac{k_{x} k_{z}}{4 \sqrt{2}} & -\frac{i k_{x} k_{y}}{4 \sqrt{2}} & -\frac{\left(k_{x}+2 i k_{y}\right) k_{z}}{4 \sqrt{6}} & 0 & 0 \\
\frac{k_{z}}{2} & \frac{k_{x}-i k_{y}}{2 \sqrt{3}} & 0 & 0 & -\frac{k_{x}-i k_{y}}{2 \sqrt{6}} & 0 \\
\frac{k_{x}+i k_{y}}{2 \sqrt{3}} & \frac{k_{z}}{6} & \frac{k_{x}-i k_{y}}{3} & 0 & \frac{k_{z}}{3 \sqrt{2}} & -\frac{k_{x}-i k_{y}}{6 \sqrt{2}} \\
0 & \frac{k_{x}+i k_{y}}{3} & -\frac{k_{z}}{6} & \frac{k_{x}-i k_{y}}{2 \sqrt{3}} & \frac{k_{x}+i k_{y}}{6 \sqrt{2}} & \frac{k_{z}}{3 \sqrt{2}} \\
0 & 0 & \frac{k_{x}+i k_{y}}{2 \sqrt{3}} & -\frac{k_{z}}{2} & 0 & \frac{k_{x}+i k_{y}}{2 \sqrt{6}} \\
-\frac{k_{x}+i k_{y}}{2 \sqrt{6}} & \frac{k_{z}}{3 \sqrt{2}} & \frac{k_{x}-i k_{y}}{6 \sqrt{2}} & 0 & \frac{k_{z}}{3} & \frac{k_{x}-i k_{y}}{3} \\
0 & -\frac{k_{x}+i k_{y}}{6 \sqrt{2}} & \frac{k_{z}}{3 \sqrt{2}} & \frac{k_{x}-i k_{y}}{2 \sqrt{6}} & \frac{k_{x}+i k_{y}}{3} & -\frac{k_{z}}{3}
\end{array}\right),
\end{aligned}
$$

Note that $M_{0-3, \boldsymbol{k}}^{(\Gamma)}$ are non-zero at $\boldsymbol{k}=0$, whereas the other gap functions are zero at $\boldsymbol{k}=0$. Considering that the Fermi sheets around $\Gamma$ is small in RhSi, only the gap functions $M_{0-3, k}^{(\Gamma)}$ are able to open a sizable superconducting gap over the Fermi sheets near $\Gamma$. Hence, around $\Gamma$, the gap function $\Delta(\boldsymbol{k})$ can be approximated as

$$
\Delta(\boldsymbol{k}) \approx\left(\Delta_{0}-\Delta_{1}\right) I_{6}+\left(\Delta_{2}-\Delta_{3}\right) \Delta_{ \pm}^{\Gamma}
$$

\section{B. Expansion of $M_{i, k}$ near the $\mathrm{R}$ point}

Writing down the first non-zero term, the Taylor expansion of $M_{i, \boldsymbol{k}}^{(\mathrm{R})} \equiv \mathbb{P}_{\mathrm{R}}^{\dagger} M_{i, \boldsymbol{k}_{\mathrm{R}}+\boldsymbol{k}} \mathbb{P}_{\mathrm{R}}$ around $\mathrm{R}$ in $\boldsymbol{k}$ is given by

$$
\begin{array}{lll}
M_{0, \boldsymbol{k}}^{(\mathrm{R})}=I_{8}, & M_{4, \boldsymbol{k}}^{(\mathrm{R})}=\Delta_{ \pm}^{\mathrm{R}}, \\
M_{1, \boldsymbol{k}}^{(\mathrm{R})}=\delta H_{1, \boldsymbol{k}}^{(\mathrm{R})}, & M_{2, \boldsymbol{k}}^{(\mathrm{R})}=\delta H_{r, 1, \boldsymbol{k}}^{(\mathrm{R})}, & M_{3, \boldsymbol{k}}^{(\mathrm{R})}=\delta H_{r, 2, \boldsymbol{k}}^{(\mathrm{R})}, \\
M_{6, \boldsymbol{k}}^{(\mathrm{R})}=\delta H_{s, 1, \boldsymbol{k}}^{(\mathrm{R})}, & M_{7, \boldsymbol{k}}^{(\mathrm{R})}=\delta H_{s, 2, \boldsymbol{k}}^{(\mathrm{R})}, & M_{8, \boldsymbol{k}}^{(\mathrm{R})}=\delta H_{s, 3, \boldsymbol{k}}^{(\mathrm{R})},
\end{array}
$$

and

$$
M_{5, \boldsymbol{k}}^{(\mathrm{R})}=-\frac{1}{2 \sqrt{2}}\left(\begin{array}{cccc}
\sqrt{2} k_{z} & k_{x}-i k_{y} & 0 & k_{x}-i k_{y} \\
k_{x}+i k_{y} & 0 & k_{x}-i k_{y} & -\sqrt{2} k_{z} \\
0 & k_{x}+i k_{y} & -\sqrt{2} k_{z} & -k_{x}-i k_{y} \\
k_{x}+i k_{y} & -\sqrt{2} k_{z} & -k_{x}+i k_{y} & 0
\end{array}\right) \oplus\left(\begin{array}{cccc}
\sqrt{2} k_{z} & k_{x}-i k_{y} & 0 & k_{x}-i k_{y} \\
k_{x}+i k_{y} & 0 & k_{x}-i k_{y} & -\sqrt{2} k_{z} \\
0 & k_{x}+i k_{y} & -\sqrt{2} k_{z} & -k_{x}-i k_{y} \\
k_{x}+i k_{y} & -\sqrt{2} k_{z} & -k_{x}+i k_{y} & 0
\end{array}\right) .
$$

Note that only $M_{0, \boldsymbol{k}}^{(\mathrm{R})}$ and $M_{4, \boldsymbol{k}}^{(\mathrm{R})}$ are non-zero at $\boldsymbol{k}=0$, and these gap functions are the decisive ones determining the sign of the gap function for each band for small $k$. Therefore, near R, we can approximate $\Delta(\boldsymbol{k})$ in Eq. (25) as

$$
\Delta(\boldsymbol{k}) \approx \Delta_{0} I_{8}+\Delta_{4} \Delta_{ \pm}^{\mathrm{R}}
$$

in the small $k$ limit. However, when the Fermi sheets are large, the typical magnitude of the Fermi momentum is too large to ignore the $\boldsymbol{k}$-dependent contributions from other $M_{i, \boldsymbol{k}}^{(\mathrm{R})}$ s. The superconducting phases with non-negligible $\Delta_{i \neq 0,4}$ 's will be discussed in Sec. V. 


\section{GENERIC DIII TOPOLOGICAL SUPERCONDUCTING PHASES WITH THE $s_{+} \oplus s_{-}$GAP FUNCTIONS}

In the main text, we focus on the topological superconducting phase with a MZM on $\bar{\Gamma}$ and nodal rings around R because $\mu_{\mathrm{R}}>E_{\mathrm{c}, 2}$ in the candidate materials such as RhSi. If the nodal rings are not present, the DIII TSC phases from $\Delta_{\Gamma(\mathrm{R})}\left(\beta_{\Gamma(\mathrm{R})}\right)$ with a higher winding number $\left|w_{3 \mathrm{D}}\right|>1$ are possible in which multiple topologically stable MZMs are guaranteed.

Table II shows all possible $w_{3 \mathrm{D}}$ in the fully gapped superconducting phases from $\Delta_{\Gamma}\left(\beta_{\Gamma}\right)$ and $\Delta_{\mathrm{R}}\left(\beta_{\mathrm{R}}\right)$ for nonzero $\alpha_{\mathrm{R}}$ assuming that $\alpha_{\Gamma}$ is positive. For a negative $\alpha_{\Gamma}$, we can obtain the corresponding winding numbers from $\left(\left|\alpha_{\Gamma}\right|,-\alpha_{\mathrm{R}}\right)$. From the table, one can find that TSC phases with a higher winding number $w_{3 \mathrm{D}}$ can be realized from $\Delta_{\Gamma(\mathrm{R})}\left(\beta_{\Gamma(\mathrm{R})}\right)$.

\begin{tabular}{|c|c|}
\hline (a) & \begin{tabular}{|c}
$\left(w_{3 \mathrm{D}, \mathrm{R}}, w_{1 \mathrm{D}, \mathrm{R}}\right)$ \\
$(-2,+1)(2,+1)$
\end{tabular} \\
\hline E $(2,+1)$ & $(0,+1) \quad(4,+1)$ \\
\hline 3 & $(-1,-1)(3,-1)$ \\
\hline$\stackrel{\text { po }}{3}(-2,+1)$ & $(-4,+1)(0,+1)$ \\
\hline
\end{tabular}

\begin{tabular}{|c|c|c|}
\hline (b) & & $\begin{array}{c}\left(w_{3 \mathrm{D}, \mathrm{R}}, w_{1 \mathrm{D}, \mathrm{R}}\right) \\
(2,+1)(-2,+1)\end{array}$ \\
\hline \multirow{3}{*}{$\begin{array}{l}\text { E } \\
\hat{5} \\
3 \\
5\end{array}$} & $(2,+1)$ & $(4,+1) \quad(0,+1)$ \\
\hline & $(1,-1)$ & $(3,-1)(-1,-1)$ \\
\hline & & $(0,+1)(-4,+1$ \\
\hline
\end{tabular}

Table II: Winding numbers $\left(w_{3 \mathrm{D}}, w_{1 \mathrm{D}}\right)$ for (a) $\alpha_{\mathrm{R}}>0$ and (b) $\alpha_{\mathrm{R}}<0$ with $\alpha_{\Gamma}>0$. Here we arrange possible $w_{3 \mathrm{D}, \Gamma}$ $\left(w_{3 \mathrm{D}, \mathrm{R}}\right)$ from the bottom to top (left to right) with increasing $\beta_{\Gamma}\left(\beta_{\mathrm{R}}\right)$.

\section{NODAL RINGS AROUND R AND THEIR STABILITY}

As studied in the main text, topological nodal rings around $\mathrm{R}$ in the BdG spectrum arise when $\Delta_{ \pm}^{\mathrm{R}}$ is dominant in $\Delta_{\mathrm{R}}\left(\beta_{\mathrm{R}}\right)$ and the Fermi level $\mu_{\mathrm{R}}$ lies above the band crossing energy level $E_{c, 2}$. In this section, we delineate the nodal rings in the BdG spectrum and their topological stability.

To construct an effective Hamiltonian for the bands crossing at $E=E_{c, 2}$, we first note that, on the $\Gamma-\mathrm{R}$ line which is invariant under $C_{3,[111]}$, the three-fold rotaion along the [111] direction, the two bands which cross at $E=E_{c, 2}$ have different eigenvalues $\lambda_{1}=-1$ and $\lambda_{2}=\exp (-i \pi / 3)$ under $C_{3,[111]}$ which protects the band crossing point. Using the wave functions for these bands as the basis vectors, the effective Hamiltonian around the crossing point is written as $[2]$

$$
H_{\mathrm{BdG}}(\boldsymbol{q})=\left(\begin{array}{cc}
H(\boldsymbol{q}) & \boldsymbol{\Delta} \\
\boldsymbol{\Delta} & -H(\boldsymbol{q})
\end{array}\right)
$$

with

$$
\begin{aligned}
H(\boldsymbol{q}) & =h_{0}(\boldsymbol{q}) \sigma_{0}+\boldsymbol{h}(\boldsymbol{q}) \cdot \boldsymbol{\sigma}, \\
h_{0}(\boldsymbol{q}) & =E_{c, 2}-\mu+c_{\|} q_{3}+c_{\perp} q_{\perp}^{2}, \\
h_{3}(\boldsymbol{q}) & =a_{\|} q_{3}+b_{\perp} q_{\perp}^{2} \\
h_{1(2)}(\boldsymbol{q}) & =a_{\perp} q_{1(2)} \\
\boldsymbol{\Delta} & =\left(\begin{array}{cc}
\Delta_{1} & 0 \\
0 & -\Delta_{2}
\end{array}\right)+O\left(q^{2}\right),
\end{aligned}
$$

where $q_{\perp}^{2}=q_{1}^{2}+q_{2}^{2}$. As shown in Fig. S2(a), we define the band crossing point as the origin of $\boldsymbol{q}=\left(q_{1}, q_{2}, q_{3}\right)$. Here, the $q_{3}$ axis is chosen along the $\Gamma-\mathrm{R}$ direction, and $q_{1}, q_{2}$ are the in-plane coordinates perpendicular to the $q_{3}$ axis. Note that $\Delta_{1} \Delta_{2}>0$ when $\Delta_{ \pm}^{(\mathrm{R})}$ is dominant in which the nodal rings can appear. Hence, from now on we assume that both $\Delta_{1}$ and $\Delta_{2}$ are positive without loss of generality. 
(a)



(b)

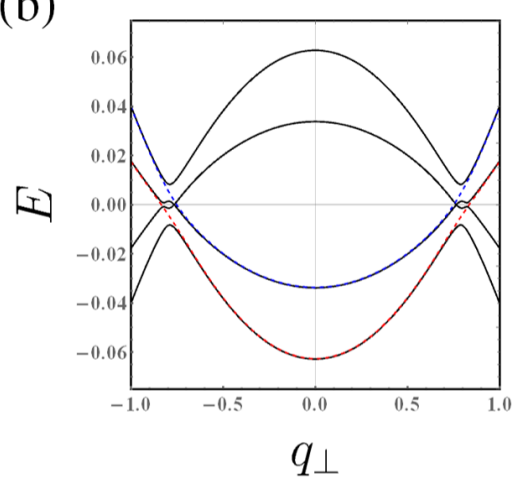

(c)

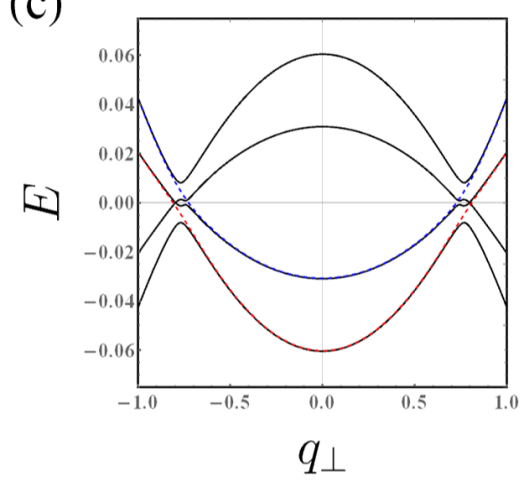

Figure S2: (a) $\left(q_{1}, q_{2}, q_{3}\right)$ coordinate system, nodal rings and the integration paths $\mathcal{L}_{n}$ in the first Brillouin zone. The blue (red) ring represents the nodal ring obtained from the solution $\boldsymbol{q}^{(1)}\left(\boldsymbol{q}^{(2)}\right)$. The green rings represent $\mathcal{L}_{n}$. (b), (c) BdG spectra at $q_{3}=q_{3}^{(1)}$ and $q_{3}=q_{3}^{(2)}$. The black lines represent the BdG spectra while the blue/red dashed lines show the two normal electronic bands crossing at $E=E_{c, 2}$.

To get an insight from the weak-pairing limit, we rewrite $H_{\mathrm{BdG}}(\boldsymbol{q})$ in the basis which diagonalizes $H(\boldsymbol{q})$ :

$$
H_{\mathrm{BdG}}(\boldsymbol{q}) \approx\left(\begin{array}{cccc}
h_{0}+h & 0 & \delta+\frac{h_{3}}{h} \bar{\Delta} & \frac{h_{1}-i h_{2}}{h} \bar{\Delta} \\
0 & h_{0}-h & \frac{h_{1}+i h_{2}}{h} \bar{\Delta} & \delta-\frac{h_{3}}{h} \bar{\Delta} \\
\delta+\frac{h_{3}}{h} \bar{\Delta} & \frac{h_{1}-i h_{2}}{h} \bar{\Delta} & -h_{0}-h & 0 \\
\frac{h_{1}+i h_{2}}{h} \bar{\Delta} & \delta-\frac{h_{3}}{h} \bar{\Delta} & 0 & -h_{0}+h
\end{array}\right),
$$

where $h=|\boldsymbol{h}|=\sqrt{h_{1}^{2}+h_{2}^{2}+h_{3}^{2}}, \bar{\Delta}=\left(\Delta_{1}+\Delta_{2}\right) / 2$, and $\delta=\left(\Delta_{1}-\Delta_{2}\right) / 2$. In the weak pairing limit where $\Delta_{1}$ and $\Delta_{2}$ are very small, the gap function for the electronic band with the dispersion $h_{0}+h$ is $\delta+\frac{h_{3}}{h} \bar{\Delta}$, which ranges from $-\Delta_{2}$ to $+\Delta_{1}$. Therefore, we could expect a region in which the BdG spectrum is gapless. Exactly the same argument is applicable to the BdG spectrum from the electronic band with the dispersion $h_{0}-h$.

To confirm the existence of the gapless excitation in the BdG spectrum, we diagonalize $H_{\mathrm{BdG}}(\boldsymbol{q})$. The eigenvalues of $H_{\mathrm{BdG}}(\boldsymbol{q})$ are given by

$$
E_{ \pm}^{2}=h_{0}^{2}+h^{2}+\bar{\Delta}^{2}+\delta^{2} \pm 2 \sqrt{\left(h_{1}^{2}+h_{2}^{2}\right)^{2}\left(h_{0}^{2}+\bar{\Delta}^{2}\right)+\left(h_{0} h_{3}+\bar{\Delta} \delta\right)^{2}} .
$$

$E_{-}^{2}(\boldsymbol{q})=0$ occurs at $\boldsymbol{q}$ satisfying

$$
h_{0}^{2}(\boldsymbol{q})-h^{2}(\boldsymbol{q})=\delta^{2}-\bar{\Delta}^{2}, \quad h_{3}(\boldsymbol{q}) \bar{\Delta}=h_{0}(\boldsymbol{q}) \delta .
$$

In the weak-pairing limit, the solutions $\boldsymbol{q}$ for the first equation form the two Fermi sheets around the band crossing point, whereas those for the second equation form paraboloids around the $q_{3}$-axis in general. Therefore, the solutions of Eq. (41) are found near the intersections between the paraboloids and the Fermi sheets.

Considering the isotropy in $\left(q_{1}, q_{2}\right)$ in $H_{\mathrm{BdG}}(\boldsymbol{q})$, we find two solutions $\boldsymbol{q}^{(1)}=\left(q_{\perp}^{(1)}, q_{3}^{(1)}\right)$ and $\boldsymbol{q}^{(2)}=\left(q_{\perp}^{(2)}, q_{3}^{(2)}\right)$ of Eq. (41):

$$
\left(q_{\perp}^{(1)}, q_{3}^{(1)}\right) \approx(0.766,-0.337), \quad\left(q_{\perp}^{(2)}, q_{3}^{(2)}\right) \approx(0.796,-0.343) .
$$

Here, the parameters $c_{\perp}=0.077, c_{\|}=-0.45, a_{\perp}=0.006, a_{\|}=-0.043, b_{\perp}=-0.024 \mathrm{eV}$ are used for RhSi, and $\Delta_{1}=0.004, \Delta_{2}=0.003$ and $E_{c, 2}-\mu=-0.2 \mathrm{eV}$ are chosen as an example.

Figures S2(b) and S2(c) show the BdG spectrum of $H_{\mathrm{BdG}}(\boldsymbol{q})$ along the $q_{\perp}$-axis at $q_{3}=q_{3}^{(1)}$ and $q_{3}=q_{3}^{(2)}$, respectively. The nodal points are clearly seen. These points actually correspond to nodal rings in Fig. S2(a).

Knowing the location of nodal rings, it is straightforward to test the stability of the nodal rings. Calculating the winding number [3] $w_{1 \mathrm{D}}^{(\mathrm{NR})}=\oint_{\mathcal{L}_{n}} \frac{\mathrm{d} \phi}{2 \pi i} \operatorname{Tr}\left[Q_{n}^{-1} \partial_{\theta} Q_{n}\right]$ along the paths $\mathcal{L}_{n}$ drawn as the green rings in the inset of Fig. $\mathrm{S} 2$ (a) where $Q_{n}(r, \theta)=H\left(q_{\perp}^{(n)}+r \cos \theta, 0, q_{3}^{(n)}+r \sin \theta\right)-i \boldsymbol{\Delta}$, we get $w_{1 \mathrm{D}}^{(\mathrm{NR})}=+1$ for $n=1$ and $w_{1 \mathrm{D}}^{(\mathrm{NR})}=-1$ for $n=2$. Thus, the nodal rings in the BdG spectrum are topologically stable. 
Two remarks are in order regarding the description of the nodal rings presented here. First, even if the Hamiltonian $H_{\mathrm{BdG}}(\boldsymbol{q})$ in Eq. (33) is valid around the band crossing point, the nodal rings could survive beyond the range of validity of $H_{\mathrm{BdG}}(\boldsymbol{q})$ due to their topological stability. Second, the argument given here is also applicable to the system with an anti-crossing between the bands. The only modification is just to add a mass term to $h_{1}(\boldsymbol{q})$ or $h_{2}(\boldsymbol{q})$ to describe the anti-crossing. This addition may affect the location of nodal rings but does not invalidate their existence.

\section{LINEARIZED GAP EQUATION}

\section{A. Calculation of the linearized gap equation}

To estimate the superconducting gap functions from the three short-ranged interactions introduced in the main text, we need to find the minimum point of the free energy $\mathcal{F}\left[\hat{\Delta}_{i}\right][4,5]$ where $\hat{\Delta}_{i}$ 's denote the pairing channels. Written up to the second order in $\hat{\Delta}_{i}$, the free energy is given by

$$
\mathcal{F}\left[\hat{\Delta}_{i}\right]=\frac{1}{2} \sum_{i} \frac{1}{U_{i}} \operatorname{Tr}\left[\hat{\Delta}_{i}^{\dagger} \hat{\Delta}_{i}\right]-\frac{k_{B} T}{2} \sum_{\boldsymbol{k}, \omega_{n}, i, j} \operatorname{Tr}\left[\hat{\Delta}_{i}^{\dagger} G_{0}\left(i \omega_{n}, \boldsymbol{k}\right) \hat{\Delta}_{j} G_{0}\left(-i \omega_{n}, \boldsymbol{k}\right)\right],
$$

where $U_{i}$ is the interaction strength for the $i$-th pairing channel $\hat{\Delta}_{i}, G_{0}$ is the Green's function for a quasi-particle in the normal phase and $\omega_{n}$ is a fermionic Matsubara frequency. To our case, the pairing channel $\hat{\Delta}_{i}$ is identified with $\Delta_{i} M_{i, \boldsymbol{k}}$ and the interaction strengths are defined as $U_{0}=U / 4, U_{1,5}=V-3 J$, and $U_{2,3,4,6,7,8}=V+J$, as stated in the main text. To find the minimum point of $\mathcal{F}\left[\hat{\Delta}_{i}\right]$, we first differentiate it with respect to $\Delta_{i}$ yielding a set of equations named the linearized gap equation (LGE). Written in the matrix form, the LGE is given by

$$
\Delta=U \chi \Delta,
$$

where $U=\operatorname{diag}\left(U_{0}, U_{1}, \ldots, U_{8}\right), \Delta=\left(\Delta_{0}, \Delta_{1}, \ldots, \Delta_{8}\right)$, and $\chi$ is the matrix of the pairing susceptibility whose elements are defined as

$$
\chi_{i j}=-k_{B} T \sum_{\omega_{n}, \boldsymbol{k}} \operatorname{Tr}\left[M_{i, \boldsymbol{k}} G_{0}\left(i \omega_{n}, \boldsymbol{k}\right) M_{j, \boldsymbol{k}} G_{0}\left(-i \omega_{n}, \boldsymbol{k}\right)\right] .
$$

Completing the summation over Matsubara frequencies, we have

$$
\chi_{i j}=-\sum_{\xi, \xi^{\prime}} \sum_{\boldsymbol{k}} \frac{\left\langle\xi, \boldsymbol{k}\left|M_{i, \boldsymbol{k}}\right| \xi^{\prime}, \boldsymbol{k}\right\rangle\left\langle\xi^{\prime}, \boldsymbol{k}\left|M_{j, \boldsymbol{k}}\right| \xi, \boldsymbol{k}\right\rangle}{\varepsilon_{\xi, \boldsymbol{k}}+\varepsilon_{\xi^{\prime}, \boldsymbol{k}}}\left[\tanh \frac{\beta \varepsilon_{\xi, \boldsymbol{k}}}{2}+\tanh \frac{\beta \varepsilon_{\xi^{\prime}, \boldsymbol{k}}}{2}\right],
$$

where $\xi, \xi^{\prime}$ are the band indices. Changing the summation over $\boldsymbol{k}$ to the integration over energy for each band, only the bands in the vicinity of the Fermi sheets contribute to $\chi_{i j}$. Thus we obtain

$$
\chi_{i j}=-\sum_{\xi} \sum_{\varepsilon_{\xi^{\prime}}=\varepsilon_{\xi}}\left[\int_{\mathrm{FS}_{\xi}} \frac{\mathrm{d}^{2} \boldsymbol{k}}{v_{\mathrm{F}}}\left\langle\xi, \boldsymbol{k}\left|M_{i, \boldsymbol{k}}\right| \xi^{\prime}, \boldsymbol{k}\right\rangle\left\langle\xi^{\prime}, \boldsymbol{k}\left|M_{j, \boldsymbol{k}}\right| \xi, \boldsymbol{k}\right\rangle\right] \log \frac{2 e^{\gamma} \beta \varepsilon_{c}}{\pi},
$$

where we assume that the system is time-reversal symmetric or inversion symmetric. Here $\gamma \sim 0.577$ is the Euler's constant, $v_{\mathrm{F}} \equiv\left\|\nabla \varepsilon_{\mathrm{F}}\right\|$ is the Fermi velocity and $\varepsilon_{c}$ is the energy cutoff for the pairing interactions, which is assumed to be the same for all bands and all interactions. By finding the solution $\Delta$ of Eq. (44) with the largest positive eigenvalue of $U \bar{\chi}$ where

$$
\bar{\chi}_{i j} \equiv-\sum_{\xi} \sum_{\varepsilon_{\xi^{\prime}}=\varepsilon_{\xi}}\left[\int_{\mathrm{FS}_{\xi}} \frac{\mathrm{d}^{2} \boldsymbol{k}}{v_{\mathrm{F}}}\left\langle\xi, \boldsymbol{k}\left|M_{i, \boldsymbol{k}}\right| \xi^{\prime}, \boldsymbol{k}\right\rangle\left\langle\xi^{\prime}, \boldsymbol{k}\left|M_{j, \boldsymbol{k}}\right| \xi, \boldsymbol{k}\right\rangle\right],
$$

we can estimate the most favorable superconducting gap function with the highest transition temperature.

For the practical calculation of $\bar{\chi}_{i j}$, we first decompose $\bar{\chi}_{i j}$ into the contributions from $\Gamma$ and $\mathrm{R}$ as $\bar{\chi}_{i j}=\bar{\chi}_{i j}^{(\Gamma)}+\bar{\chi}_{i j}^{(\mathrm{R})}$ and make two approximations. First, we replace the integration over the Fermi sheet $\int_{\mathrm{FS}_{\xi}} \mathrm{d}^{2} \boldsymbol{k}$ by $\int \mathrm{d} \theta \mathrm{d} \phi k_{\mathrm{F}}^{2}(\theta, \phi) \sin \theta$ since the shape of the Fermi sheets around $\Gamma$ and $R$ looks very close to that of a sphere. Small distortion from the sphere is taken into account by allowing the Fermi momentum $k_{\mathrm{F}}$ to be a function of $\theta$ and $\phi$. Second, we ignore 
the interband pairings, which is acceptable in the weak pairing limit. One could concern with the fact that there are symmetry-protected double degeneracy on the $k_{x, y, z}=\pi$ planes as the energy difference between degenerate bands is smaller than the magnitude of superconducting gap. However, the intersections between the Fermi sheets and those planes are just one-dimensional loops, whose contribution from the interband pairings to the surface integral over the Fermi sheets is usually negligible. These approximations lead to the following simplified formulas:

$$
\begin{aligned}
& \bar{\chi}_{i j}^{(\Gamma)}=-\sum_{\xi \in\{\text { in,out }\}}\left[\int_{\mathrm{FS}_{\xi}} \mathrm{d} \theta \mathrm{d} \phi \frac{k_{\mathrm{F}, \Gamma}^{2}(\theta, \phi) \sin \theta}{v_{\mathrm{F}, \Gamma}(\theta, \phi)}\left\langle\xi, \boldsymbol{k}\left|M_{i, \boldsymbol{k}}\right| \xi, \boldsymbol{k}\right\rangle\left\langle\xi, \boldsymbol{k}\left|M_{j, \boldsymbol{k}}\right| \xi, \boldsymbol{k}\right\rangle\right], \\
& \bar{\chi}_{i j}^{(\mathrm{R})}=-\sum_{\xi \in\{1,2,3,4\}}\left[\int_{\mathrm{FS}_{\xi}} \mathrm{d} \theta \mathrm{d} \phi \frac{k_{\mathrm{F}, \mathrm{R}}^{2}(\theta, \phi) \sin \theta}{v_{\mathrm{F}, \mathrm{R}}(\theta, \phi)}\left\langle\xi, \boldsymbol{k}\left|M_{i, \boldsymbol{k}}\right| \xi, \boldsymbol{k}\right\rangle\left\langle\xi, \boldsymbol{k}\left|M_{j, \boldsymbol{k}}\right| \xi, \boldsymbol{k}\right\rangle\right],
\end{aligned}
$$

where $k_{\mathrm{F}, \Gamma(\mathrm{R})}$ is the magnitude of the Fermi momentum and $v_{\mathrm{F}, \Gamma(\mathrm{R})}$ is the Fermi velocity at the Fermi sheets around $\Gamma(\mathrm{R})$. For the numerical evaluation, each numerical surface integration is done on $\theta \times \phi$ with uniform $100 \times 200$ grid points.

\section{B. $\Delta_{ \pm}, I_{6(4)}$ and other superconducting gap functions}

Solving the LGE, we get a solution as a mixture of $\Delta_{0-8}$ which should be translated as $\Delta(\boldsymbol{k})=\sum_{i} \Delta_{i} M_{i, \boldsymbol{k}}$. Note that $M_{0-4, \boldsymbol{k}}$ are even under $\boldsymbol{k} \rightarrow-\boldsymbol{k}$ and constant at $\Gamma$ or $\mathrm{R}$, while $M_{5-8, \boldsymbol{k}}$ are odd under $\boldsymbol{k} \rightarrow-\boldsymbol{k}$ whose leading order terms are linear in $\boldsymbol{k}$ around $\Gamma$ and R. According to the parity under $\boldsymbol{k} \rightarrow-\boldsymbol{k}$, we call $M_{0-4, \boldsymbol{k}}$ and $M_{5-8, \boldsymbol{k}}$ the even-parity pairings and the odd-parity pairings, respectively. In the main text, we focus on the effects of $\Delta_{ \pm}^{\Gamma(\mathrm{R})}$, thus only the even-parity parings that are reduced to either $I_{6(4)}$ or $\Delta_{ \pm}^{\Gamma(\mathrm{R})}$ are considered, while the contribution from the odd-parity gap functions is neglected. To test the validity of neglecting the odd-parity gap functions, we examine our numerical results obtained from the LGE in detail by checking the surface Green's function at every grid point in the phase diagram of Fig. 3(a) in the main text.

Figure S3 summarizes our results. Figure S3(a) is the phase diagram in Fig. 3(a) in the main text over which four representative points are marked by red dots. Here the dashed regions represent the deviation from Fig. 3(a) where the solution of the LGE is found to be not consistent with the description in the main text using only $I_{6(4)}$ and $\Delta_{ \pm}^{\Gamma(\mathrm{R})}$. The representative surface spectral weights from the surface Green's function for the "Trivial" (beige), "NR+MZM" (blue), and "NR" (red) regions are displayed in Figs. S3(b), S3(c), and S3(d), respectively. Figure S3(e) shows the surface spectral weight at the point (e) in Fig. S3(a) as a representative point in the dashed regions in Fig. S3(a). In those regions, the overall magnitude of $\sum_{i=5-8} \Delta_{i} M_{i, \boldsymbol{k}}$ at the Fermi sheets is not quite small compared to that of $\sum_{i=0-4} \Delta_{i} M_{i, \boldsymbol{k}}$. The non-negligible odd-parity gap functions $\sum_{i=5-8} \Delta_{i} M_{i, \boldsymbol{k}}$ give rise to the unexpected nodal rings around $\mathrm{R}$ in Fig. S3(e). However, those regions are narrow and lie around the boundary between the three major regions ("Trivial", "No SC" and "NR+MZM"). Therefore, we conclude that the cases in which the odd-parity gap functions play the major role are very limited, and thus it is sufficient to consider the even-parity gap functions.

\section{Ambiguity of SOC parameters and their effect on the superconducting gap functions}

So far, our discussion is based on the result from the tight-binding model with $v_{s, i}=0$. However, as we discussed in Sec. IA, there is an ambiguity in choosing the $v_{s, i}$ 's in the tight-binding model. The presence of non-zero $v_{s, i}$ may distort the spin texture on the Fermi sheets, and the pairing susceptibility $\chi$ is influenced by these parameters. Though the topological nature does not change much by the changes of $v_{s, i}$, the magnitude of the odd-parity pairings can. These changes in the odd parity pairings whose leading order terms are linear in $\boldsymbol{k}$ near the $\Gamma$ and $\mathrm{R}$ points can induce deviations from the phase described by $\Delta_{ \pm}$and $I_{6(4)}$ which are constants in the leading order in $\boldsymbol{k}$.

Figure S4 shows the surface Green's function calculations for $v_{s, 1}=0,0.04,-0.04 \mathrm{eV}$ with $v_{s, 2}=v_{s, 3}=0$ as examples. As we change the SOC parameters, additional NRs can be found, as indicated by the red arrows in Figs. S4(b) and S4(c). Nonetheless, comparing Fig. S4(a) with Figs. S4(b) and S4(c), the topological features remain unaltered such as the MZM at the $\bar{\Gamma}$ point and the NRs around the R point. 
(a)
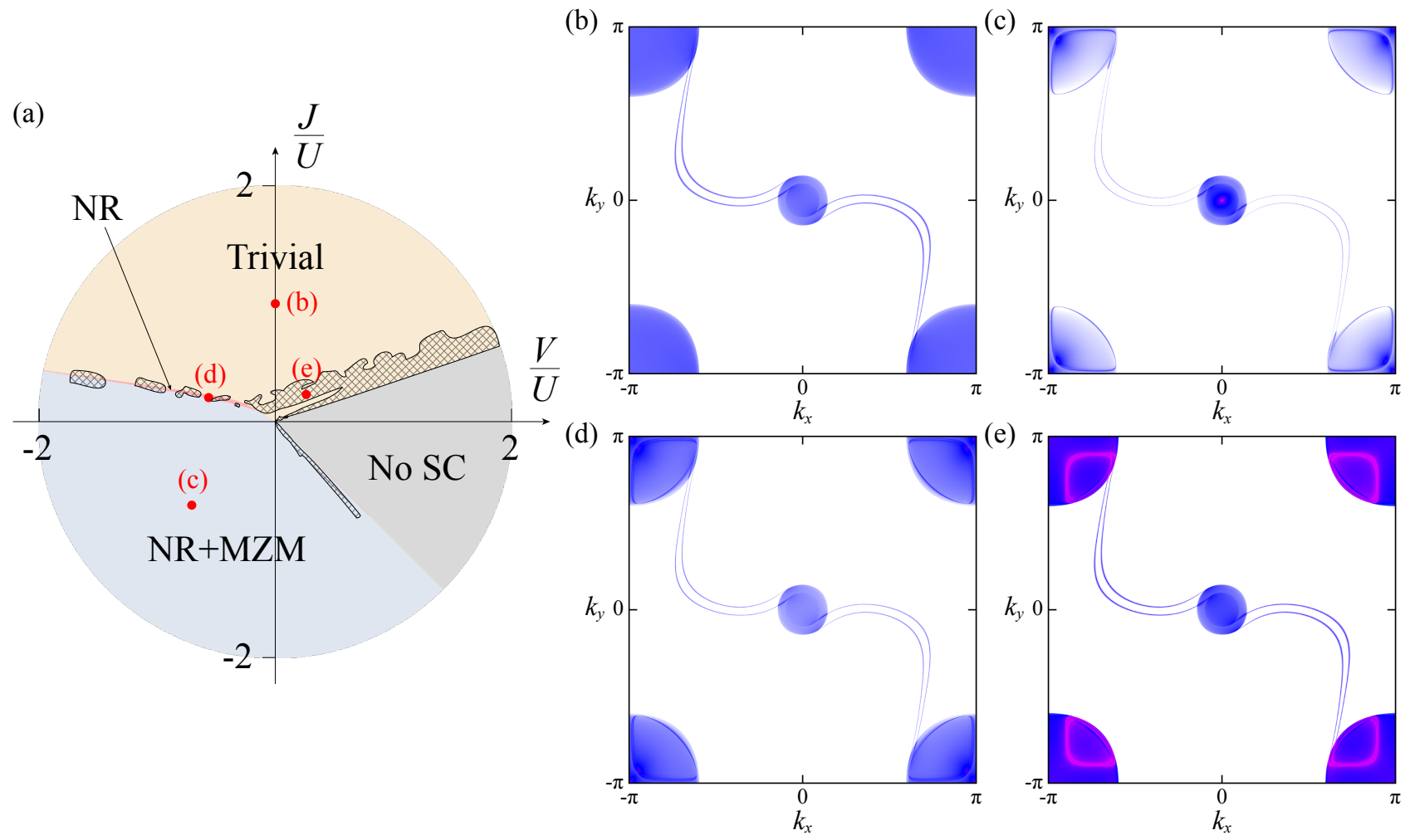

Figure S3: Phase diagram drawn by examining the surface Green's function. The dashed regions represent the deviation from Fig. 3(a) in the main text. In (b)-(e), the representative surface spectral weights from the surface Green's function calculation are displayed.


Figure S4: Surface Green's functions when $v_{s, 1}$ is (a) 0 , (b) $0.04 \mathrm{eV}$, (c) $-0.04 \mathrm{eV}$ with $\mu=1.3 m_{\Gamma}, U=1$, and $V=J=-\frac{1}{5 \sqrt{2}}$. Additional NRs are found at (b) R and (c) $\Gamma$.

[1] G. Chang, S.-Y. Xu, B. J. Wieder, D. S. Sanchez, S.-M. Huang, I. Belopolski, T.-R. Chang, S. Zhang, A. Bansil, H. Lin, and M. Z. Hasan, Phys. Rev. Lett. 119, 206401 (2017).

[2] C. Fang, M. J. Gilbert, X. Dai, and B. A. Bernevig, Phys. Rev. Lett. 108, 266802 (2012).

[3] A. P. Schnyder and S. Ryu, Phys. Rev. B 84, 060504 (2011).

[4] E. Bauer and M. Sigrist, eds., Non-Centrosymmetric Superconductors (Springer Berlin Heidelberg, 2012).

[5] H. G. Suh, H. Menke, P. M. R. Brydon, C. Timm, A. Ramires, and D. F. Agterberg, Phys. Rev. Research 2, 032023(R) (2020). 